Review

\title{
Fluoroalkyl Amino Reagents (FARs): A General Approach towards the Synthesis of Heterocyclic Compounds Bearing Emergent Fluorinated Substituents
}

\author{
Bruno Commare ${ }^{1}$, Etienne Schmitt ${ }^{1}$, Fallia Aribi ${ }^{1}$, Armen Panossian ${ }^{1}$, Jean-Pierre Vors ${ }^{2}$, \\ Sergiy Pazenok ${ }^{3}$ and Frédéric R. Leroux ${ }^{1, *}$ \\ 1 University of Strasbourg, CNRS, LCM UMR 7509, 67000 Strasbourg, France; bcommare@unistra.fr (B.C.); \\ schmitt_etienne@msn.com (E.S.); fallia.aribi@gmail.com (F.A.); armen.panossian@unistra.fr (A.P.) \\ 2 Bayer S.A.S., 14 Impasse Pierre Baizet, BP 99163, 69263 Lyon CEDEX 09, France; jean-pierre.vors@bayer.com \\ 3 Bayer AG, Alfred-Nobel-Strasse 50,40789 Monheim, Germany; sergiy.pazenok@bayer.com \\ * Correspondence: frederic.leroux@unistra.fr
}

Academic Editor: Thierry Billard

Received: 20 May 2017; Accepted: 7 June 2017; Published: 12 June 2017

\begin{abstract}
Fluorinated heterocycles are important building blocks in pharmaceutical, agrochemical and material sciences. Therefore, organofluorine chemistry has witnessed high interest in the development of efficient methods for the introduction of emergent fluorinated substituents (EFS) onto heterocycles. In this context, fluoroalkyl amino reagents (FARs) - a class of chemicals that was slightly forgotten over the last decades-has emerged again recently and proved to be a powerful tool for the introduction of various fluorinated groups onto (hetero)aromatic derivatives.
\end{abstract}

Keywords: fluorine; FAR; heterocycles; fluoroalkyl; difluoromethyl; emergent fluorinated substituents

\section{Introduction}

The incorporation of fluorine or fluorinated moieties into organic compounds plays a key role in life science-oriented research, as it can often result in profound changes to the physico-chemical and biological properties of the resulting compounds [1]. Therefore, organofluorine chemistry has become a new challenge in the context of small-molecule research in agro- [2-8] and medicinal chemistry [9-13]. Consequently, extensive and increasing attention has been devoted in the last decades to the development of new and more efficient methods for the introduction of fluorinated motifs. Classic methods for rapid assembly of fluoralkyl-substituted compounds rely almost exclusively on the commercial availability of fluorinated building blocks that are manufactured by Swarts-type reactions, a method for which no industrially viable substitute existed up to recently. Indeed, an alternative strategy emerged in the last decade in industrial scale applications, based on the use of fluoroalkyl amino reagents (FARs) as new tools to introduce fluoroalkyl moieties. This review will cover the preparation and the reactivity of FARs as well as their numerous applications.

\section{Preparation and Properties of Fluoroalkyl Amino Reagents}

\subsection{Preparation and Availability}

Following the discovery of polytetrafluoroethylene (PTFE) by Plunkett in 1938, early examples of $N, N$-dialkyl $\alpha, \alpha$-difluoroalkylamines made from fluorinated alkenes were reported right after the Second World War. Indeed, the first reaction between nucleophiles and chlorotrifluoroethylene was reported for the first time in 1950 by Pruett et al. [14]. Then, Knunyants et al. reported in 1956 the addition of several 
nucleophiles, including secondary amines, on perfluoropropene [15]. In 1959, Yarovenko et al. described for the first time the preparation and application of 2-chloro- $N, N$-diethyl-1,1,2-trifluoroethan-1-amine (1b), later called the Yarovenko reagent, for deoxyfluorination of alcohols [16]. In 1960, England et al. reported a broad extension of the scope of a number of base-catalyzed additions to fluoro-olefins [17]. Although already synthesized by the Knunyants group, Ishikawa et al. described in 1979 the preparation of $\mathrm{N}, \mathrm{N}$-diethyl-1,1,2,3,3,3-hexafluoropropan-1-amine (1c) by condensation of perfluoropropene and diethylamine [18]. Based on previous work from the England group, Petrov et al. described completely the preparation of 1,1,2,2-tetrafluoro- $N, N$-dimethylethan-1-amine (1a, TFEDMA, sometimes called Petrov's reagent) in 2001 [19]. Recently Walkowiak et al. reported the preparation of other FARs from 1,1,3,3,3-pentafluoropropene and various secondary amines to study the influence of alkyl chains of the secondary amine on the HF elimination process [20].

Nowadays, Petrov's reagent (1a), Yarovenko's reagent $(\mathbf{1 b})$ and Ishikawa's reagent (1c) are commercially available from many suppliers, but their syntheses remain unchanged. FARs are still prepared by hydroamination of polyfluoroalkenes with secondary amines, which are both bulk chemicals produced on ton-scale in the fluoropolymer industry. This represents an advantage, as both ingredients for the preparation of FARs are rather cheap. TFEDMA (the Petrov reagent) can be purchased in a relatively high purity ( $>97 \% \mathrm{wt}$.) and the use of this yellow liquid is very convenient. Yarovenko's reagent is a dark brown oil (available with 97\% wt. purity), whereas the Ishikawa reagent is a pale brown oil with lower purity (ca. $90 \% \mathrm{wt}$.). Both are less stable than TFEDMA and degrade much more rapidly. Their purity must be measured prior to use by means of NMR analysis in strictly anhydrous, non-protic and non-nucleophilic deuterated solvents (e.g., $\left.\mathrm{CD}_{3} \mathrm{CN}\right)$. One should indeed have always in mind that these FARs have to be handled under inert-gas atmospheres, as they are moisture sensitive, and their hydrolysis results in the release of hydrofluoric acid (HF). In 2015 a new FAR, $\left(\mathrm{CF}_{3} \mathrm{OCFHCF} \mathrm{N}_{2} \mathrm{~N}\left(\mathrm{CH}_{3}\right)_{2}\right) \mathbf{1} \mathbf{d}$ was developed by Leroux and Pazenok $[21,22]$ for the introduction of $\mathrm{CHFOCF}_{3}$ as a challenging emergent fluoroalkyl substituent. It can be prepared in situ under its activated form (see next section) from commercially available gaseous trifluoromethyl trifluorovinyl ether. The new fluoroalkoxyfluoroalkyl group is highly electron withdrawing and has lower steric hindrance than $\mathrm{CHFCF}_{3}$ (in Ishikawa's reagent) due to the oxygen spacer between the $\mathrm{CF}_{3}$ moiety and the reactive electrophilic center (Scheme 1).<smiles>FC(F)=C(F)F</smiles>

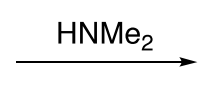<smiles>CN(C)C(F)(F)C(F)F</smiles><smiles>FC(F)=C(F)Cl</smiles><smiles>CCNCC</smiles><smiles>CCN(CC)C(F)(F)C(F)Cl</smiles>

1b

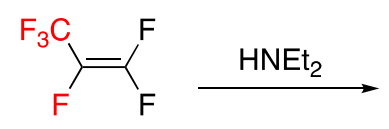<smiles>CCN(CC)C(F)(F)C(F)C(F)(F)F</smiles>

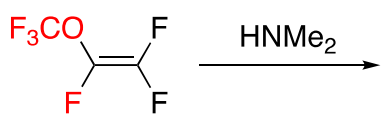

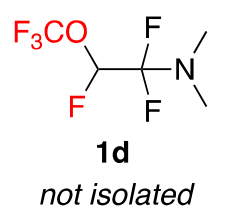

Petrov et al. 2001

Yarovenko et al. 1959

Ishikawa et al. 1979

Leroux-Pazenok et al. 2015

Scheme 1. Preparation of fluoroalkyl amino reagents (FARs)—hydroamination of polyfluoroalkenes. 


\subsection{Lewis Acid Activation of Fluoroalkyl Amino Reagents}

FARs show a unique reactivity due to the presence of highly electron-withdrawing fluorine atoms located closely to the tertiary amine. Indeed, the negative hyperconjugation resulting from an overlap of the filled non-bonding orbital of nitrogen with an empty anti-bonding orbital of the C-F bond weakens the latter to generate an equilibrium between the amines $\mathbf{1 a}-\mathbf{d}$ and the fluoroiminium forms $\mathbf{2 a}-\mathbf{d}$, although intermediates $\mathbf{2 a - d}$ could never be observed directly. This phenomenon is responsible for the specific reactivity of FARs. The difluoroalkylamine/fluoroiminium equilibrium can be fully shifted to the iminium form after activation by a Lewis acid, yielding iminium tetrafluoroborate salts $\mathbf{3 a}-\mathbf{d}$ in case of $\mathrm{BF}_{3} \cdot \mathrm{OEt}_{2}$. These intermediates display a powerful electrophilic reactivity similarly to an acylium ion (Scheme 2). They also have some structural analogy with well-known iminium salts, such as the Vilsmeier reagent [23].

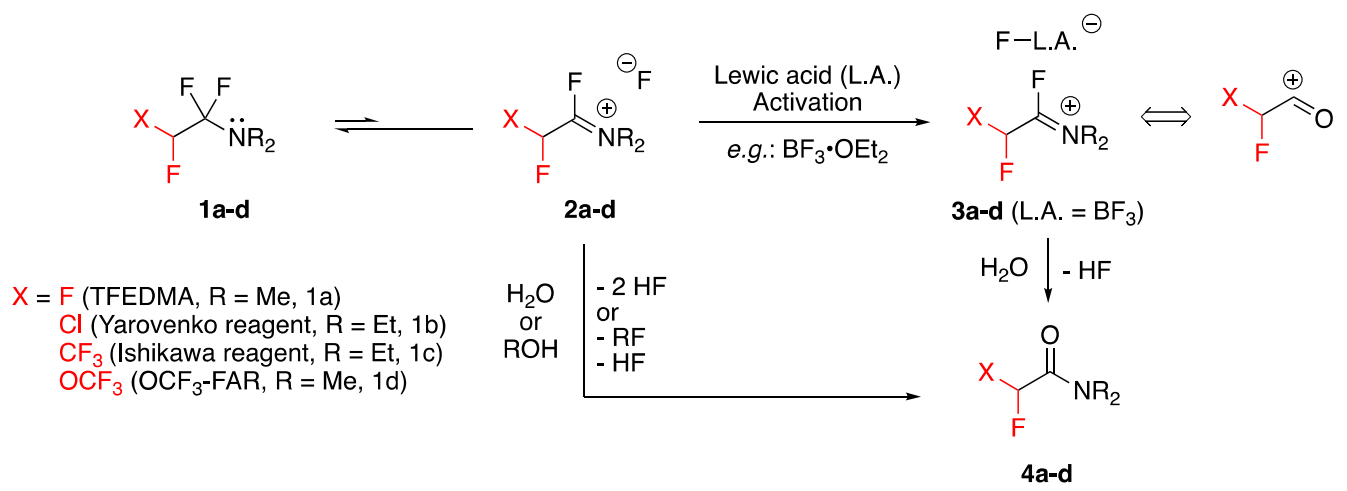

Scheme 2. Lewis acid-mediated activation of FARs. TFEDMA, 1,1,2,2-tetrafluoro- $N, N$-dimethylethan-1-amine.

Both fluoroiminiums (fluoride $\mathbf{2 a -} \mathbf{d}$ or tetrafluoroborate $\mathbf{3 a}-\mathbf{d}$ salts) are highly moisture sensitive; they release hydrogen fluoride in contact with air to afford the corresponding fluorinated acetamides 4a-d. The activation of FARs with Lewis acids is usually carried out in DCM or MeCN. The activated form is soluble in $\mathrm{MeCN}$ whereas it precipitates in DCM; evaporation of the latter solvent allows to isolate the fluoroiminium salt which is stable for a few hours under inert atmosphere (only for a few minutes under air). TFEDMA $1 \mathrm{a}$ and "OCF $3-\mathrm{FAR}^{\prime} 1 \mathrm{~d}$ can be used quite conveniently without this precipitation step; thus, they can be activated directly in $\mathrm{MeCN}$ over $15 \mathrm{~min}$, as this solvent usually constitutes the medium for further reactions. However, due to their lower purity and slower activity, the Yarovenko $\mathbf{1 b}$ and Ishikawa $1 \mathrm{c}$ reagents are activated over $45 \mathrm{~min}$ to $1 \mathrm{~h}$ and are preferably used after precipitation from DCM (Scheme 3).

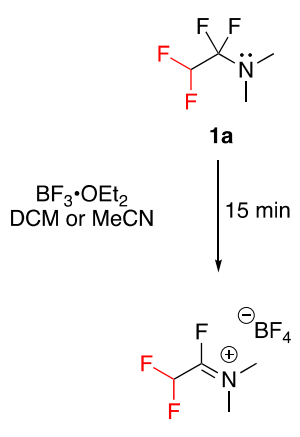

3a

soluble in $\mathrm{MeCN}$ white precipitate (DCM)
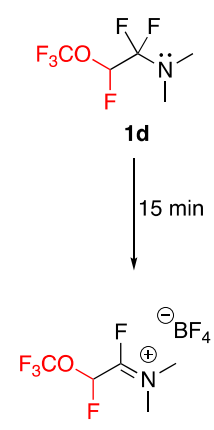

3d

soluble in $\mathrm{MeCN}$ white precipitate (DCM)

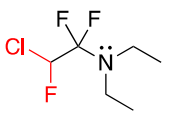

$1 b$
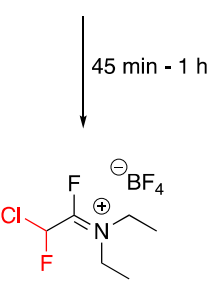

3b

soluble in $\mathrm{MeCN}$ brown solid (DCM)
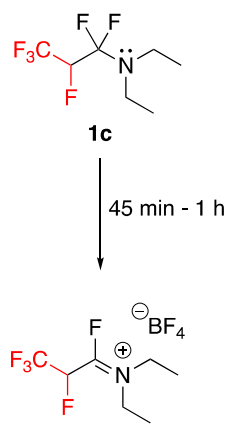

$3 c$

soluble in $\mathrm{MeCN}$ brown solid (DCM)

Scheme 3. Activation of FARs with $\mathrm{BF}_{3} \cdot \mathrm{OEt}_{2}$. 
Concerning the choice of the Lewis acid, boron trifluoride diethyl etherate $\left(\mathrm{BF}_{3} \cdot \mathrm{OEt}_{2}\right)$ and aluminium (III) chloride are commonly used with a preference for the first one. Indeed, the activation of TFEDMA with $\mathrm{AlCl}_{3}$ in $\mathrm{MeCN}$ is slightly longer than with $\mathrm{BF}_{3} \cdot \mathrm{OEt}_{2}$ ( $1 \mathrm{~h}$ instead of $<15 \mathrm{~min}$ ). The resulting counter-anion is also important in the reactivity of FARs. Tetrahedral $\mathrm{BF}_{4}{ }^{-}$is less nucleophilic and basic than nitrates and halides and tetrafluoroborate salts are usually more soluble in organic solvents. Experimentally, FARs are usually rather simple to use on small-scale reactions even though small quantities of hydrogen fluoride are released. Simple glassware was conveniently used without excessive corrosion. Teflon flasks are however used when reactions are carried out on large scale ( $>10$ g scale).

\section{Fluoroalkyl Amino Reagents: Efficient Tools for Fluorination and for the Transfer of Fluoroalkyl Groups}

\subsection{General Reactivity Modes of FARs}

The high electrophilicity of FARs, especially in their activated iminium form, and their ability to release hydrogen fluoride confer them a specific reactivity, which can be divided in four modes (Scheme 4) depending on the substrates and on the FAR used:

(A) No carbon of the FAR is incorporated in the desired product of the reaction. The FAR acts as an activator of hydroxyl groups, leading to their replacement by fluorine (with release of the hydrolysed FAR as a fluorinated acetamide) or another intramolecular nucleophile as in an example of Beckmann rearrangement. Aldehydes can also be deoxofluorinated. (Section 3.2).

(B) All carbons of the FAR are present in the desired product of the reaction but only one, the carbon of the iminium, undergoes transformations via one or two nucleophilic attack(s). This reactivity mode concerns the acylation of aromatic derivatives (Section 3.3.) and the synthesis of fluorinated heterocycles by ring-closing attacks of heteroatomic nucleophiles (Section 3.4).

(C) All carbons of the FAR are present in the desired product of the reaction and 2 carbons, the carbon of the iminium and the methine in $\alpha$ position, undergo transformations. This kind of reactivity is observed when nucleophiles are either allylic or propargylic alcohols (Section 3.5).

(D) All carbons of the FAR are present in the desired product of the reaction and all of them, namely the carbon of the iminium, the $\alpha$-methine and the carbon in $\beta$ position $\left(\mathrm{CF}_{3}\right)$ undergo transformations. Accordingly, this reactivity is observed only with the Ishikawa reagent (Section 3.6).

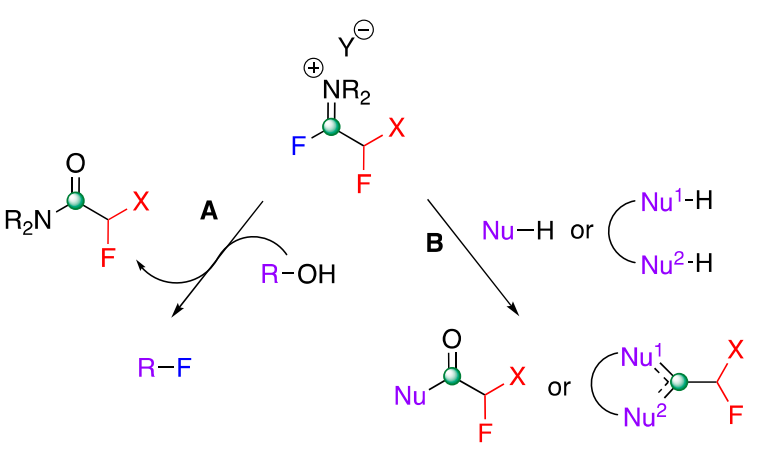

$$
\begin{gathered}
\mathrm{X}=\mathrm{F}, \mathrm{Cl}, \mathrm{CF}_{3} \text { or } \mathrm{OCF}_{3} \\
\mathrm{Y}=\mathrm{F}, \mathrm{BF}_{4}, \mathrm{AlCl}_{3} \mathrm{~F} \\
\mathrm{R}=\mathrm{Me} \text { or } \mathrm{Et}
\end{gathered}
$$
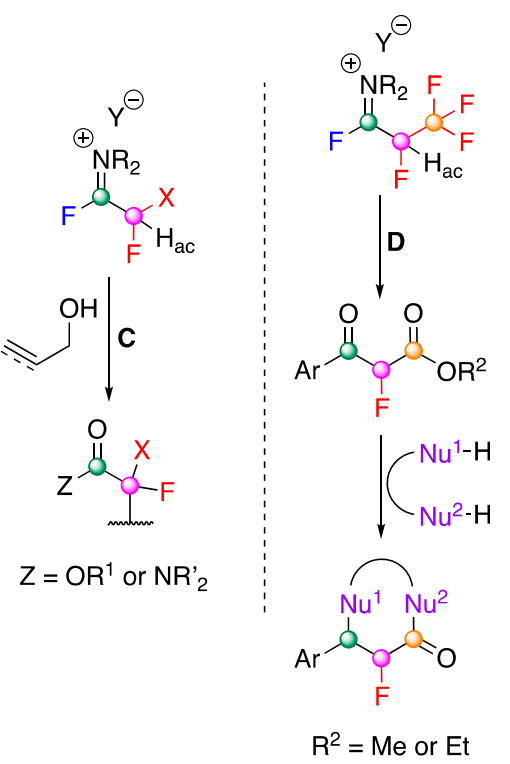

Scheme 4. Overview of the diverse reactivity modes and applications of FARs. 


\subsection{Nucleophilic Fluorination of the Hydroxyl or Carbonyl Functions}

Since the 1960s till today, the Petrov reagent (1a), the Yarovenko reagent (1b) and the Ishikawa reagent (1c) have been commonly used as selective fluorination agents of compounds containing a hydroxyl moiety, such as alcohols [18,19,24-49], including hydroxyproline [50-53] or carbohydrate derivatives [54], sulfonic [19] and carboxylic acids [55-58]. Interestingly, carbonyl compounds can also react with FARs to afford difluoromethylated compounds [59]. The mechanism consists in the formation of intermediate 6 as a result of the reaction between the hydroxyl function and the fluoroiminium followed by the decomposition of intermediate 6 to afford the fluorinated product 7 and the corresponding fluorinated acetamide $\mathbf{4 a - c}$ (Scheme 5).

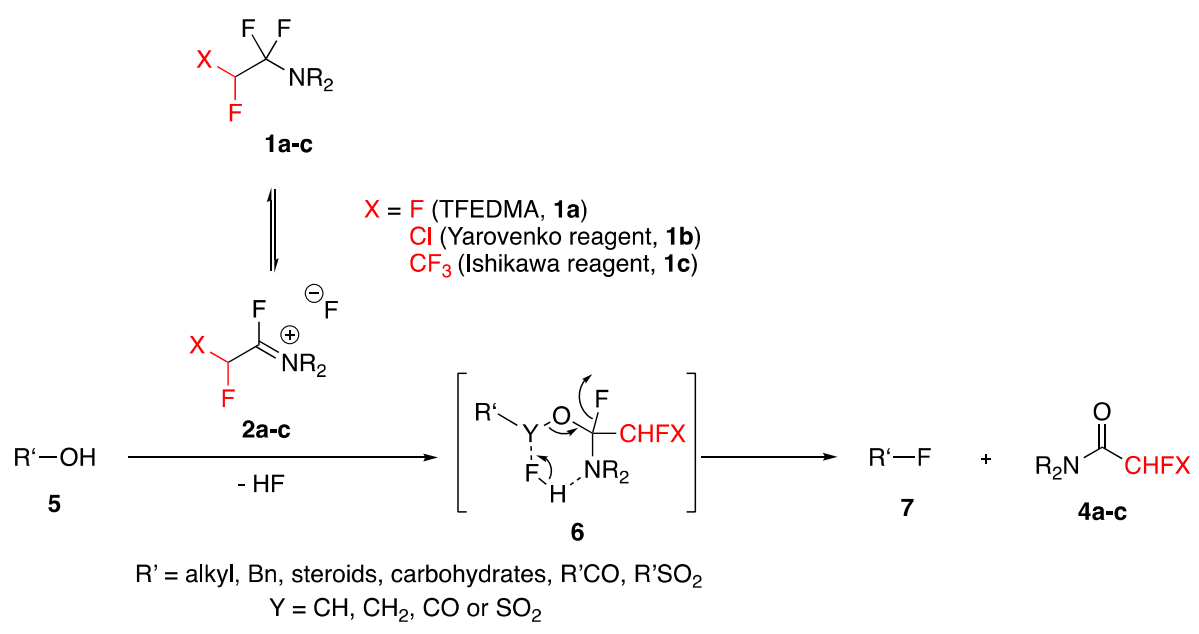

Scheme 5. Dehydroxyfluorination — mechanism proposed by Petrov et al. [19].

The Yarovenko [60] and the Ishikawa [61] reagents were also used to prepare amide compounds thanks to their capacity to provide efficiently acyl fluorides from carboxylic acids. They have also found applications as dehydrating agents to prepare acetylenic ketones from $\beta$-diketones [62]. TFEDMA can be reacted with 1,3-linear diketones (enolizable ketones) to provide $\beta$-difluoroketones [63].

Finally, the Yarovenko reagent was also used to trigger the Beckmann rearrangement of $\alpha$-methioxyketoxime (Scheme 6) [64,65]. Indeed, the hydroxylamine can react with the Yarovenko reagent to form intermediate 9 . Then, instead of undergoing an attack by the fluoride anion, as for usual reactions of alcohols with FARs, intermediate $\mathbf{1 0}$ engages in an intramolecular addition of the sulfur atom, releasing the fluorinated acetamide $4 \mathrm{~b}$ and leading to a thiazete $\mathbf{1 1}$ which finally fragments.

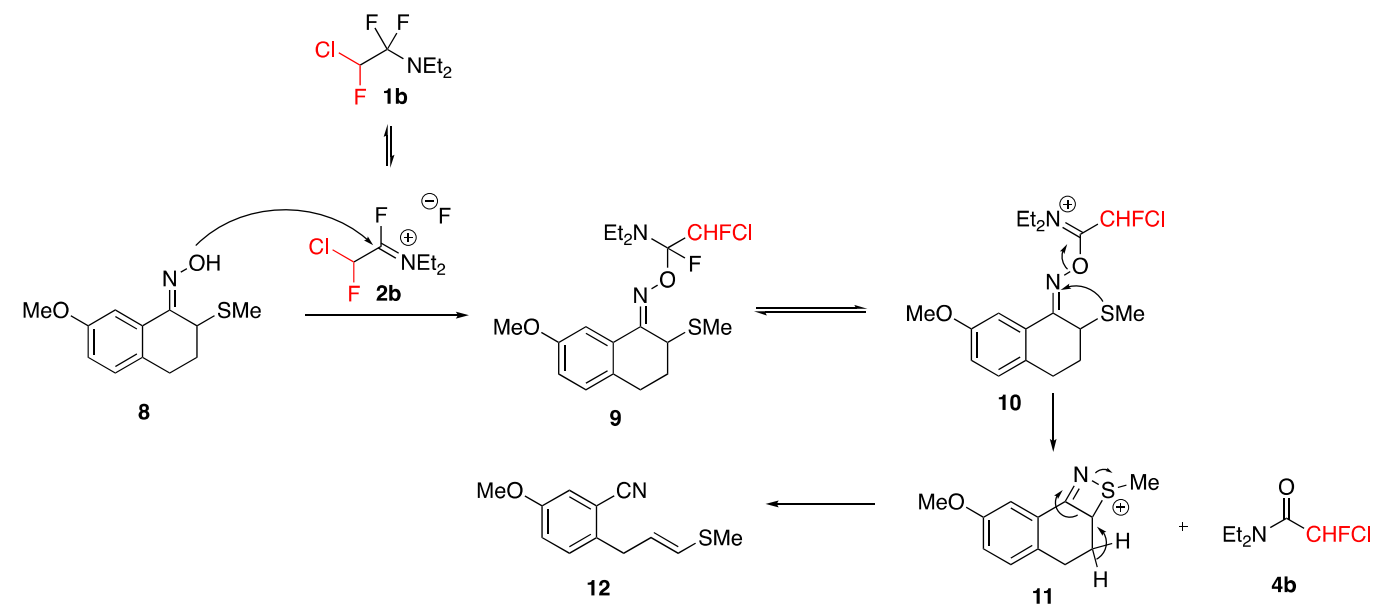

Scheme 6. Beckmann rearrangement initiated by the Yarovenko reagent. 


\subsection{Acylation of Aromatics}

The chemistry of FARs enriched in 1975, when Wakselman et al. used them for the fluoroacylation of electron-rich aromatics and more precisely, of dimethylaminobenzene, naphthalene, indole, thiophene and N-Me-pyrrole (compounds 13a-j) using the activated forms of TFEDMA (3a), Yarovenko's reagent (3b) and Ishikawa's reagent (3c) in a Friedel-Crafts-type reaction. After hydrolysis of the resulting arylcarbiminium salt intermediate, acylated aromatics $\mathbf{1 4 a} \mathbf{a} \mathbf{j}$ were isolated in moderate to good yields (Scheme 7) [66].

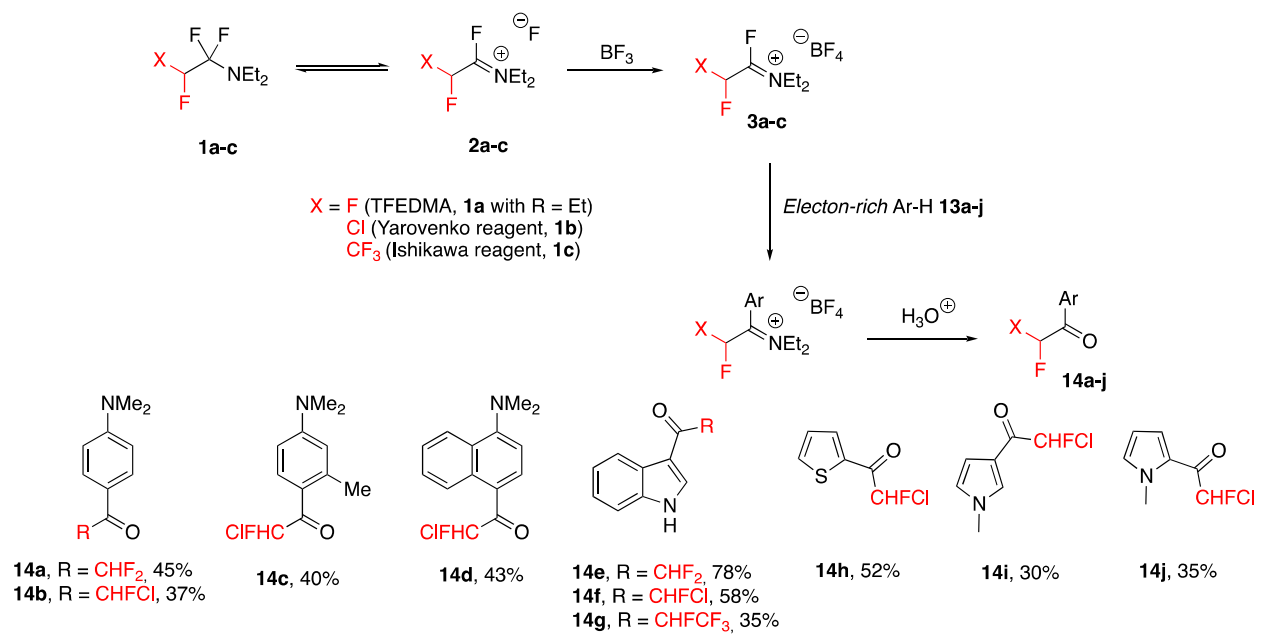

Scheme 7. Acylation of electron-rich aromatics with FARs by Wakselman et al. [66].

This method was recently extended to other heterocycles, such as pyrrole, furan, thiophene or $\mathrm{N}$-methylindole (15a-g). For example, pyrrole was efficiently difluoroacylated and the introduction of a second difluoroacyl group was achieved to provide $16 \mathrm{~b}$ with high yield. Furan $16 \mathrm{c}$ and thiophene 16d gave lower yields, due to a high volatility and sensitivity towards hydrolysis or decomposition. 3-Aminopyrazole reacted via nucleophilic attack of its most nucleophilic position, namely the amino function, to afford the corresponding amide 16e. $\mathrm{N}$-methylindole and trimethylmethyleneindoline led to the corresponding derivatives $\mathbf{1 6 f}$ and $\mathbf{1 6 g}$, respectively (Scheme 8) [67].

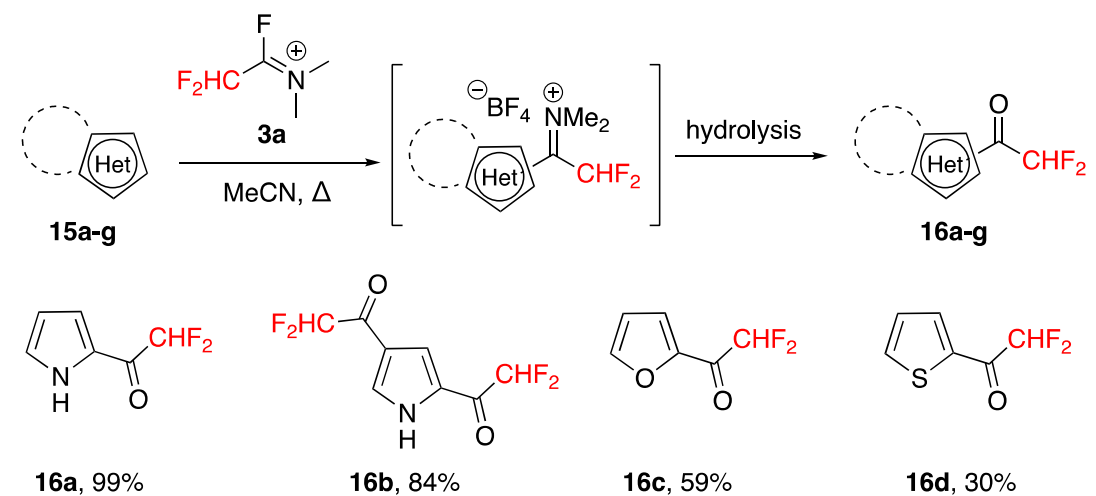<smiles>O=C(Nc1cc[nH]n1)C(F)F</smiles>

$16 e, 55 \%$<smiles>Cn1cc(C(=O)C(F)F)c2ccccc21</smiles>

$16 f, 76 \%$<smiles>Cc1cc(C(F)F)cc(=O)n1C</smiles>

$16 g, 99 \%$

Scheme 8. Difluoroacylation of electron-rich heterocycles with TFEDMA. 
Although thermal conditions usually used give good results, microwave assistance allows one to achieve much shorter reaction times and higher yields. Several difluoroacylated aniline and anisole derivatives 18a-e could be isolated with moderate to excellent yields and with a regioselectivity governed by the substituents, as in usual $S_{E} A r$ (electrophilic aromatic substitution) reactions (Scheme 9) [67].

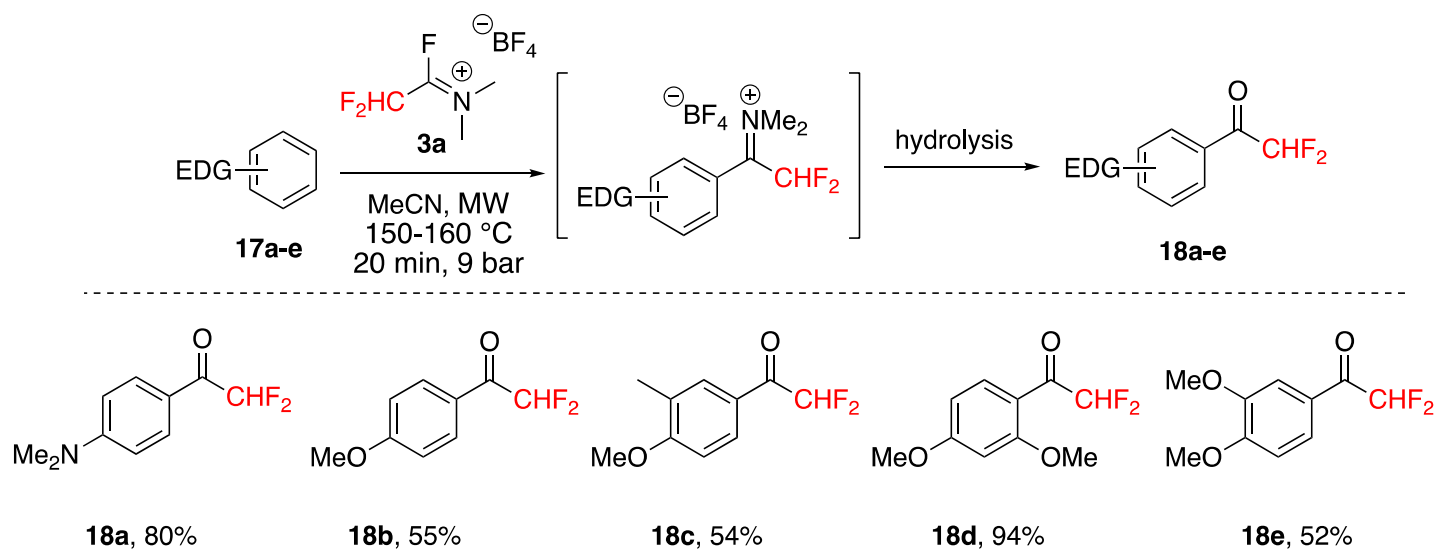

Scheme 9. Difluoroacylation of electron-rich arenes under microwave heating. EDG, electron-donating group; MW, microwave.

In order to access the other regioisomers and to broaden the substrate scope, halogen/metal exchanges can be employed to convert aryl halides into the desired difluoroacyl derivatives. Indeed, nucleophilic species formed in situ after the bromine/lithium exchange can be trapped with $\mathrm{N}, \mathrm{N}$-dimethyldifluoroacetamide (4a, obtained by hydrolysis of TFEDMA) to afford the difluoroacylated carbocycles 20a-c (Scheme 10) [67].

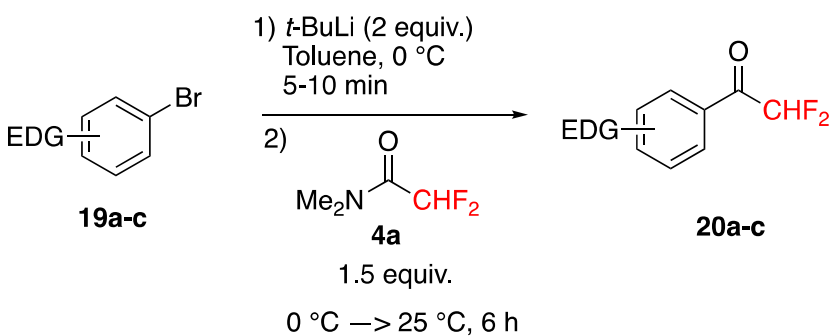

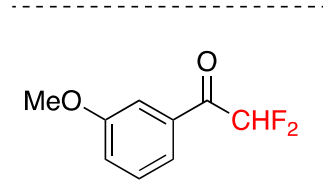

20 a, $38 \%$<smiles>COc1cc(OC)cc(C(=O)C(F)F)c1</smiles>

20b, $63 \%$<smiles>COc1cc(C(=O)C(F)F)cc(OC)c1OC</smiles>

20c, $70 \%$

Scheme 10. Difluoroacylation of electron-rich arenes with non- $S_{E} A r$ (electrophilic aromatic substitution) regioselectivity by $\mathrm{Br} / \mathrm{Li}$ exchange followed by trapping with a difluoroacetamide.

A few examples of C-fluoroacylation of non-aromatic substrates were also reported. Whereas non-cyclic 1,3-diketones undergo deoxofluorination (Section 3.2), the cyclic analogues react with TFEDMA to yield the product of fluoroacylation of the active methylene [63]. Finally, we can notice that in presence of a tertiary amine ( $N, N$-diisopropylethylamine (DIPEA) for example), some alkyl alcohols react with the Ishikawa reagent to afford the corresponding $\alpha$-perfluoroesters, i.e., the products of O-acylation instead of the usual dehydroxyfluorination [68]. Similar results were obtained with aliphatic $\beta$-nitroalcohols [69] and $\alpha$-halogenocyclohexanols [70], even in absence of additional base. 


\subsection{Synthesis of Fluoroalkylated Heterocycles}

3.4.1. Synthesis of Mono-Fluoroalkylated Benzo-Fused Heterocycles from 1,2-Diheteroatom-functionalized Arenes

In 1979, the group of Ishikawa described the first FAR-based preparation of fluoroalkylated heterocycles, such as benzimidazoles, benzothiazoles and quinazolones from the Yarovenko reagent $\mathbf{1 b}$. New heteroarene compounds $\mathbf{2 1 a}-\mathbf{j}$ bearing a $\mathrm{CHFCl}$ group are produced with yields ranging from 50 to $75 \%$ (Scheme 11) [71].

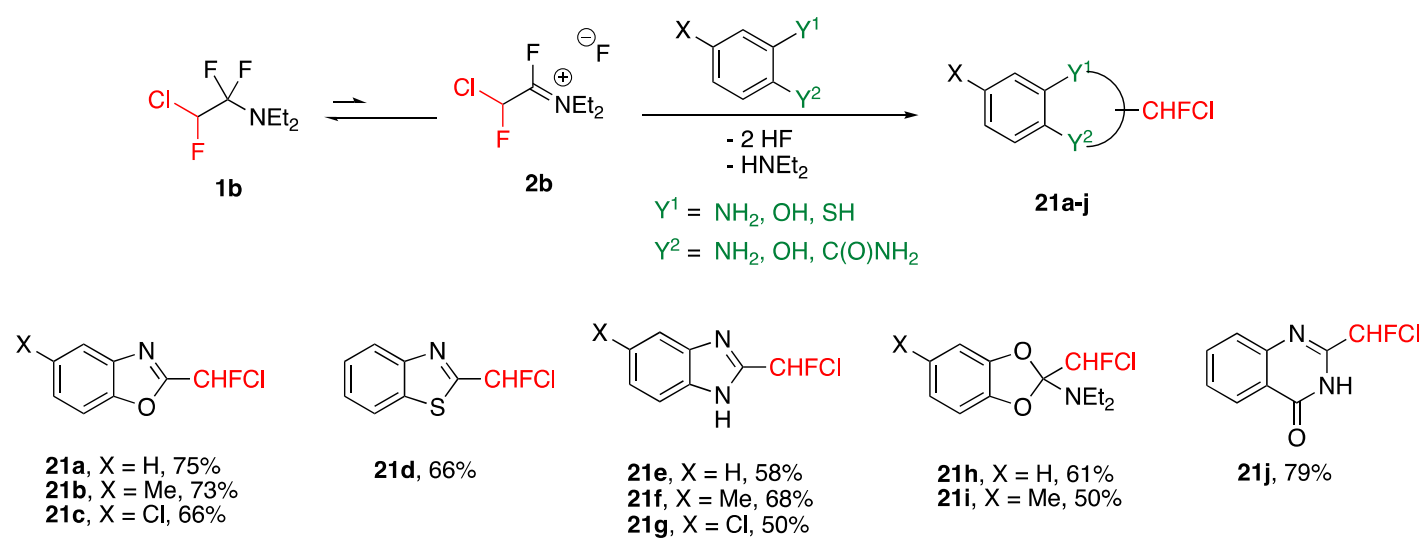

Scheme 11. Synthesis of fluoroalkylated benzo-fused heterocycles without activation of FARs by Ishikawa et al. [71].

These first results demonstrate the powerful potential of FARs to transfer fluoralkyl groups and access to various fluorinated (hetero)arenes which are ubiquitous in life science-oriented research.

\subsubsection{Synthesis of Mono-Fluoroalkylated Pyrazoles}

Since the beginning of the 21st century, difluoromethylpyrazoles [72] have attracted considerable attention in crop science, since the $3-\mathrm{CHF}_{2}$-pyrazolecarboxamide motif is actually found in new-generation top selling succinate dehydrogenase inhibitor (SDHI) fungicides (Figure 1) [72-77].

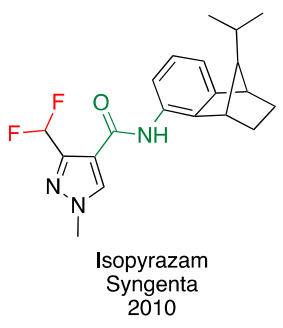

Figure 1.

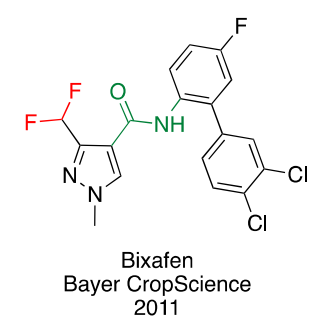

Launched succinate

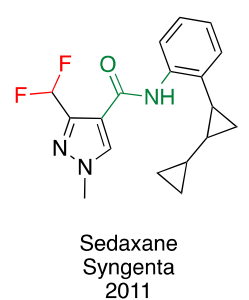

dehydrogenase

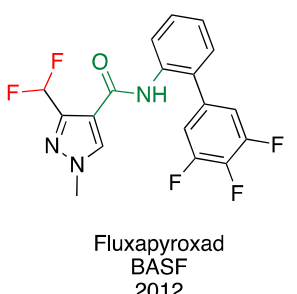

inhibitors

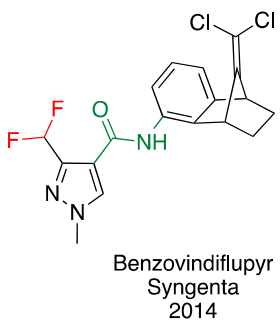

2014

fluorinated pyrazolecarboxamides.

Whereas synthetics approaches towards pyrazoles bearing "classical" fluorinated substituents ( $\mathrm{F}$ and $\mathrm{CF}_{3}$ ) have been widely studied and reviewed by Fustero et al. [78], the introduction of fluoroalkyl groups other than $\mathrm{CF}_{3}$ onto various $\mathrm{N}$-based heterocycles is still the focus of intense research interest. In 2008, Pazenok et al. reported the utilization of TFEDMA for the preparation of ethyl 3-(difluoromethyl)-1-methyl-1H-pyrazole-4-carboxylate (DFMMP), the key intermediate of Bixafen ${ }^{\circledR}$ (a modern SDHI fungicide) [79]. This first example of use of a FAR to access fluoroalkylpyrazoles prompted further investigation on FAR chemistry, as a means to develop new synthetic methods towards $\mathrm{N}$-based heterocyles bearing emergent fluorinated substituents (EFS). 
Towards the 3- $\mathrm{CHF}_{2}$-Pyrazolecarboxamide Motif

Several methods are described in the literature to prepare fluoroalkylpyrazoles. Most of them consist in the use of fluorinated precursors derived from difluoroacetic acid and subsequent cyclisation with hydrazines. All these methods were already reviewed in 2013 [80]. Another way consists in the construction of the fluoroalkyl group on the already formed pyrazole ring, by nucleophilic fluorination of chloroakyl or formyl groups or reductive dechlorination of chlorofluoroalkyl groups [81]. The first preparation of the desired DFMMP intermediate 22a (Scheme 12) was patented in 1992 and was carried out starting from ethyl difluoroacetoacetate [82]. The product was obtained with good yield $(74 \%)$, but the lack of regioselectivity and the difficult access to the starting material at this time (its availability is easier now) were major drawbacks of this first attempt. Several approaches have been described later to optimize the synthesis of DFMMP with full regioselectivity, high yield, low cost or non-toxic conditions which may be applied industrially. However, it was difficult to combine all these parameters.

To meet all required specifications, a new strategy was employed, based on the use of a specific FAR, namely TFEDMA (1a). The initial attempt involved the nucleophilic attack of ethyl 3-methoxyacrylate on activated TFEDMA to form the resulting iminium in situ, which was further cyclized by treatment with methyl hydrazine to afford the targeted DFMMP with 68\% yield and a 87:13 ratio of isomers (Scheme 12A) [83]. This partial regioselectivity can be explained by the competition of two electrophilic centers during the attack by the hydrazine, resulting from the delocalization of the positive charge along the conjugated system. The ratio could be improved to $92: 8$ by replacing ethyl $\beta$-methoxyacrylate by ethyl $\beta$-dimethylaminoacrylate (Scheme 12B) [79]. Finally, full regioselectivity and high yield $(94 \%)$ were obtained when the in situ formed fluoroiminium tetrafluoroborate salt was reacted with the protected hydrazine analogue of ethyl $\beta$-dimethylaminoacrylate (Scheme 12C) [84]. The preparations of $\mathrm{CF}_{3} \mathrm{CHF}$ - and $\mathrm{CHFCl}$-functionalized analogues were successfully carried out using the same strategy (yields are not reported).

A)

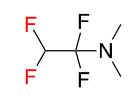

$1 a$

B)

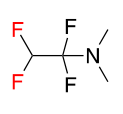

$1 a$

C)

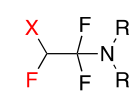

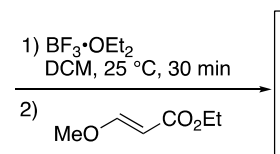

$\mathrm{MeCN}, 70^{\circ} \mathrm{C}, 21 \mathrm{~h}$

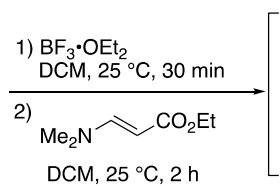

$\mathrm{DCM}, 25^{\circ} \mathrm{C}, 2 \mathrm{~h}$

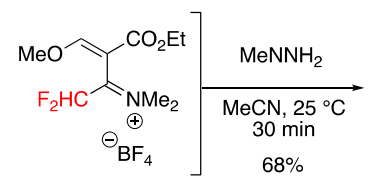<smiles>CCOC(=O)c1cn(C)nc1C(F)F</smiles><smiles>CCOC(=O)c1cnn(C)c1C(F)F</smiles>

22a $87: 13 \quad$ 23a

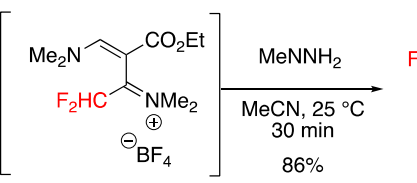

$86 \%$

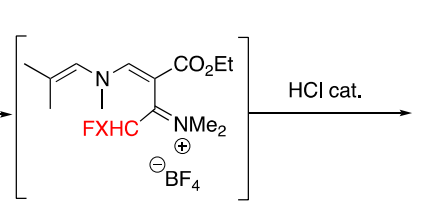

$\mathrm{MeCN}, 25^{\circ} \mathrm{C}, 1.5 \mathrm{~h}$

$\mathrm{X}=\mathrm{F}($ TFEDMA, 1a, $\mathrm{R}=\mathrm{Me})$

$\mathrm{Cl}$ (Yarovenko reagent, $\mathbf{1 b}, \mathrm{R}=\mathrm{Et})$

$\mathrm{CF}_{3}$ (Ishikawa reagent, $1 \mathrm{c}, \mathrm{R}=\mathrm{Et}$ )

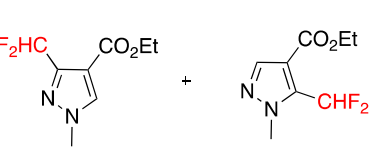

22a $92: 8 \quad$ 23a

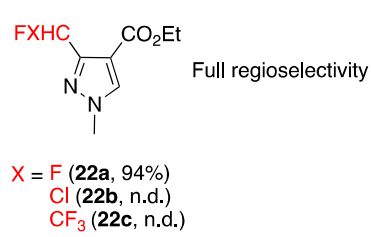

Scheme 12. Use of FARs ((A) Reaction of TFEDMA with methoxy acrylate; (B) with dimethylamino acrylate; (C) with a methylhydrazone) and acrylates to prepare ethyl 3-(difluoromethyl)-1-methyl$1 H$-pyrazole-4-carboxylate (DFMMP) with full regioselectivity.

Synthesis of Various Substituted Mono (Fluoroalkyl)pyrazoles and Isoxazoles

As described above, activated FARs reacted well with amino- or alkoxyacrylates to form in situ highly reactive dielectrophilic species, precursors of mono(fluoroalkyl)pyrazoles. As a logical extension, the reactivity towards other nucleophiles was studied to prepare several substituted mono(fluoroalkyl)pyrazoles and -isoxazoles [67]. First, activated TFEDMA 3a can react smoothly 
with vinyl ethers $\mathbf{2 4}$ and ketene acetals 28 to form iminium intermediates $\mathbf{2 5}$ and $\mathbf{2 9}$ and afford corresponding substituted mono $\left(\mathrm{CHF}_{2}\right)-\mathrm{NMe}$-pyrazoles $\mathbf{2 6 , 2 7}$ and $\mathbf{3 0}$ after cyclization with methyl hydrazine (Scheme 13).

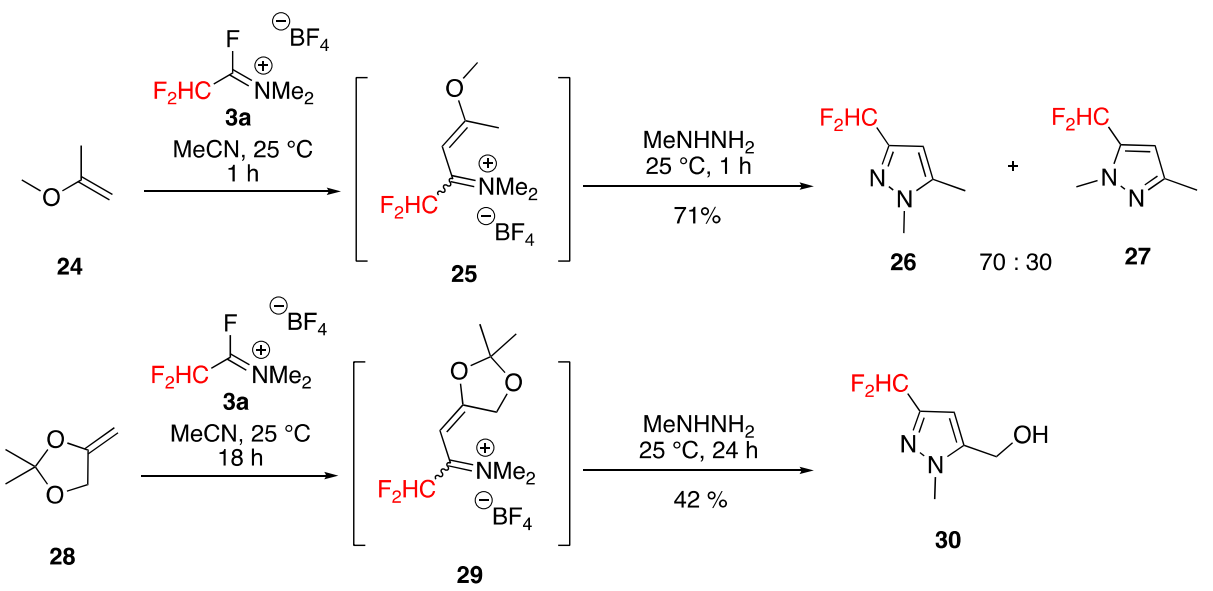

Scheme 13. Reaction of activated TFEDMA 3a with vinyl ethers.

Second, investigations about the reactivity of 3a with silyl enol ethers were conducted [67]. Commercial silyl enol ethers of cyclopentanone 31 and cyclohexanone 32 can react with fluoroiminium salt 3a affording $\mathrm{CHF}_{2}$-iminium intermediates 33 and 34, which can be either used directly in cyclization or hydrolyzed to isolate the corresponding $\beta$-(2,2-difluoro-1-hydroxy-ethylidene)cycloalkyl ketones 39 and $\mathbf{4 0}$. When treated with methyl hydrazine, 40 gave a 1:1 mixture of regioisomers $\mathbf{4 1 / 4 2}$, whereas iminium intermediates 33 and 34 led to the major isomers 35 and 37 with very good to complete regioselectivity (Scheme 14). This difference of regioselectivity can be explained by the higher electrophilicity of the iminium carbon in 33 and 34 with regard to the same carbon of enolic type in 39 and $\mathbf{4 0}$ and to the carbonyl group.

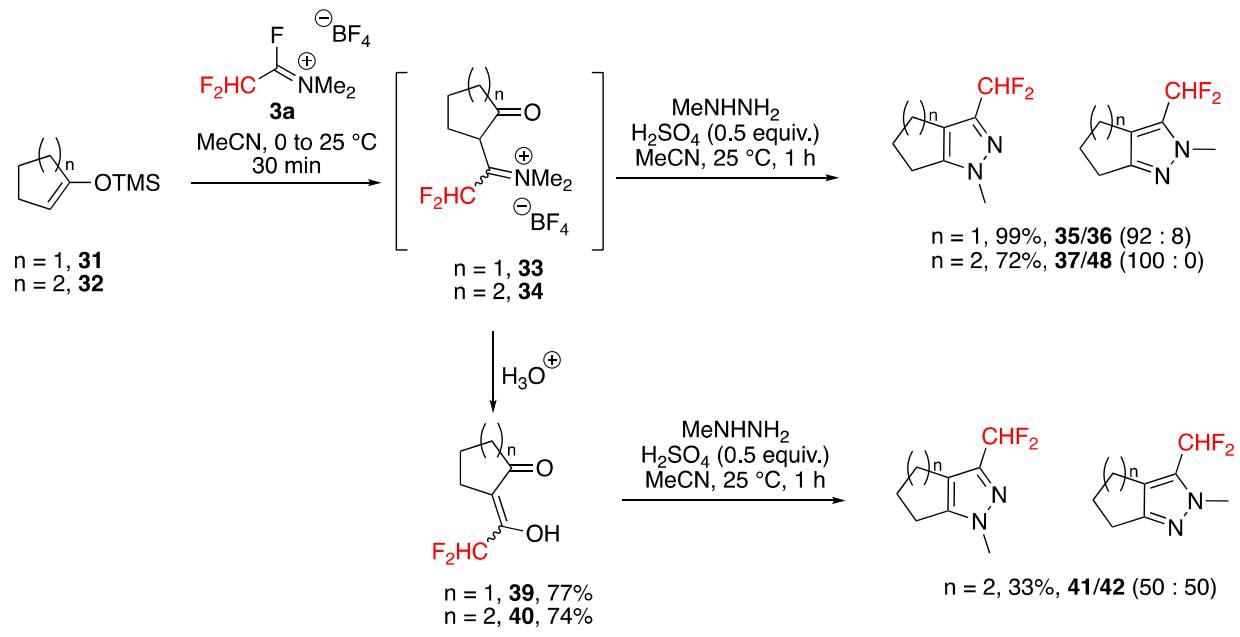

Scheme 14. Reaction of 3a with silyl enol ethers of cyclopentanone and cyclohexanone.

These differences of regioselectivity were equally observed with the silyl enol ether of acetophenone $\mathbf{4 3}$ affording the major isomer $\mathbf{4 5}$ with very good selectivity (94:6) when avoiding hydrolysis of the iminium intermediate 44 . The latter was able to react also with hydrazine hydrate and hydroxylamine hydrochloride to provide $\mathrm{NH}$-pyrazole 47 and isoxazole 48 respectively. The silyl enol ether of acetylacetone 50 afforded a single acetyl pyrazole isomer 52 (Scheme 15) [67]. 


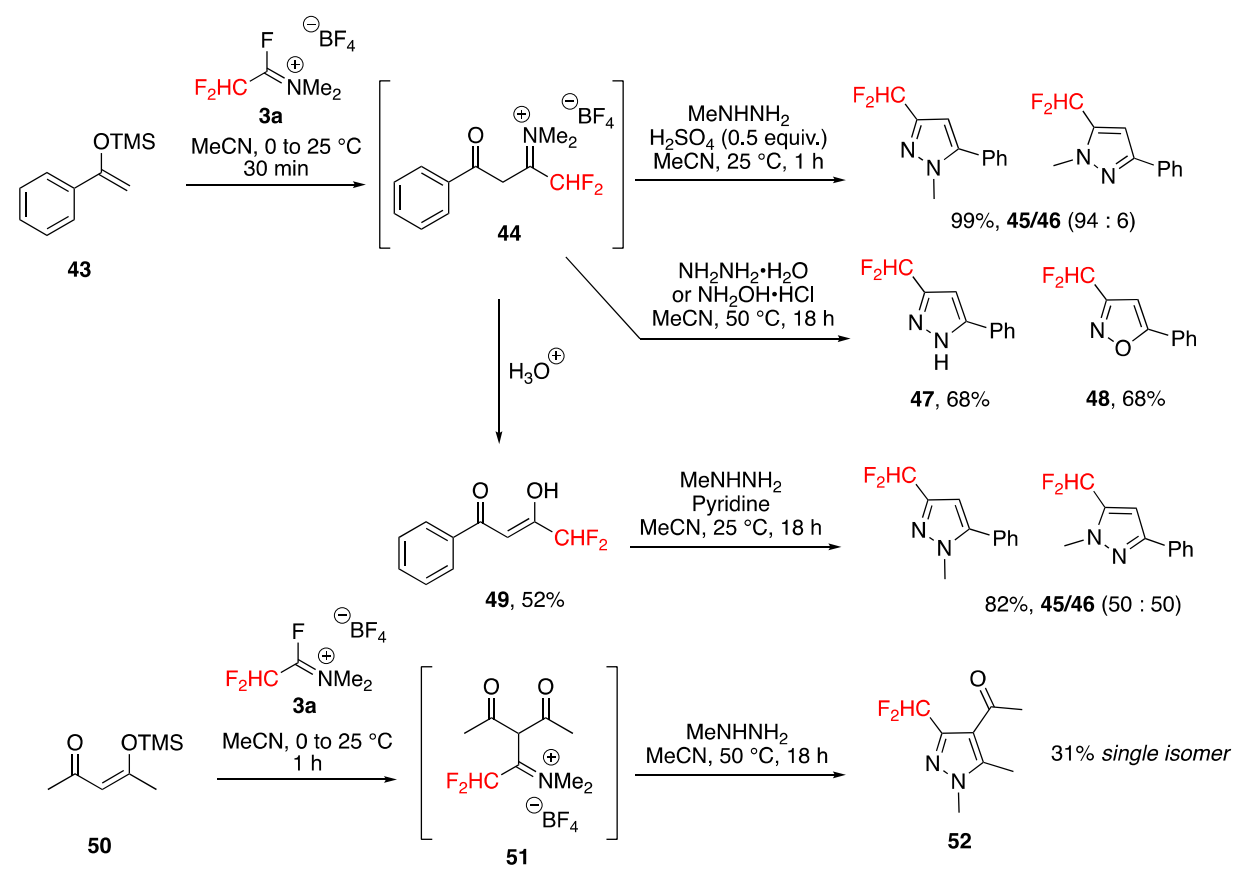

Scheme 15. Reaction of 3a with silyl enol ethers of acetophenone and acetylacetone.

Third, 3-difluoromethylpyrazoles and -isoxazoles bearing an amino group in position 5 can be obtained by reacting activated TFEDMA 3a and $\mathrm{CH}$-acidic nitrile derivatives, namely malononitrile 53 and ethyl cyanoacetate 58 , and following with a cyclization step with hydrazines or hydroxylamine (Scheme 16). The implementation of the first stage of the reaction proved delicate. The choice of the base and the isolation of the intermediate difluoro(dimethyamino)ethylidenes $\mathbf{5 4}$ and 59 appeared critical. However, the cyclization step was much easier and afforded efficiently 3-difluoromethyl-5-aminopyrazoles (55, 56, 60 and 61) and -isoxazoles (57 and 62) in presence of corresponding dinucleophiles (BOC-hydrazide (BOC, tert-butoxycarbonyle) was used instead of hydrazine hydrate in the case of compound $\mathbf{6 1}$ in order to improve the efficacy of the reaction) [67]. Last, monofluoroalkylpyrazoles could also be prepared by reaction of activated FARs and azines; this strategy will be described in Synthesis of 3,5-Bis(fluoroalkyl)-NH-pyrazoles from Azines Section.

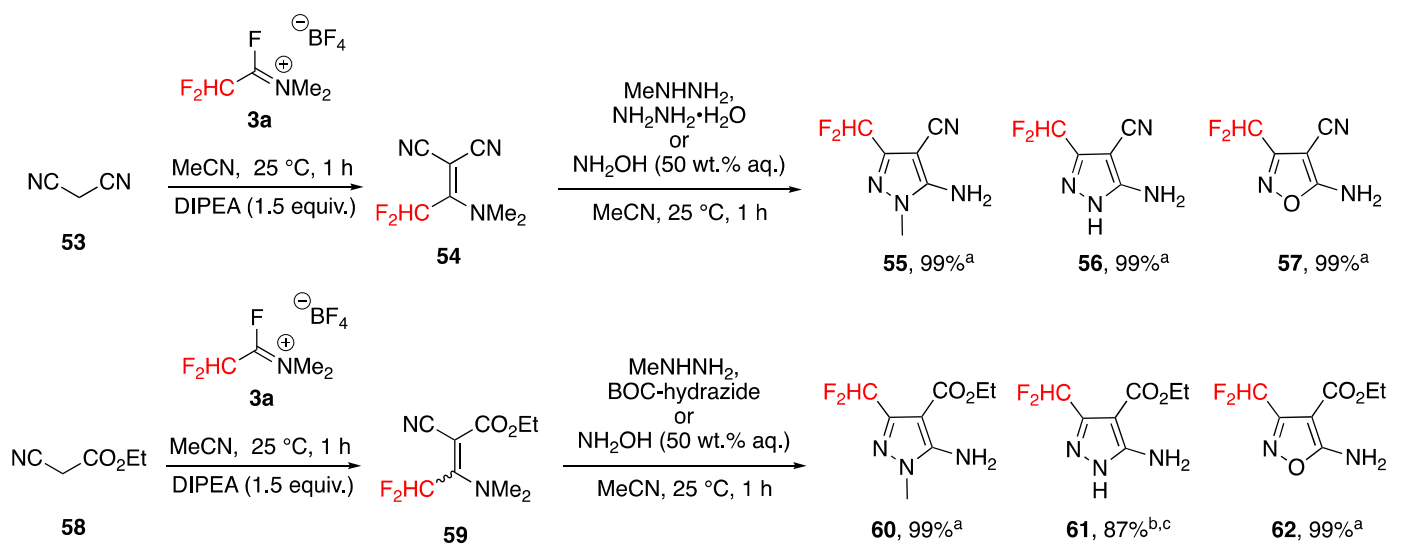

Scheme 16. Preparation of difluoromethyl 5-aminopyrazoles- and isoxazoles. a ${ }^{19} \mathrm{~F}-\mathrm{NMR}$ yield using $\mathrm{PhF}$ as internal standard. ${ }^{\mathrm{b}}$ isolated yield. ${ }^{\mathrm{c}} 40{ }^{\circ} \mathrm{C}, 18 \mathrm{~h}$. DIPEA, $\mathrm{N}, \mathrm{N}$-diisopropylethylamine. 


\subsubsection{Synthesis of Bis-fluoroalkylated Pyrazoles}

The huge diversity of targets in crop science and the success of DFMMP derivatives (Figure 1) motivated the search for analogues of this key motif bearing an additional fluoroalkyl group on the pyrazole ring.

Synthesis of 3,5-Bis(fluoroalkyl)pyrazoles from Fluoroacetoacetates

Previous work already described the synthesis of pyrazoles bearing two fluorinated groups by reaction of bisperfluoroalkyl diketones with hydrazines, but the synthesis, isolation and purification of the starting fluorinated diketones is very complex [85-90]. To circumvent these issues, FARs proved a very valuable tool and allowed to develop a scalable and operationally convenient method. Indeed, they could act as a source of one fluoroalkyl group, while the other one was provided by available fluoroacetoacetates, leading after treatment with hydrazines to 3,5-bis(fluoroalkyl)-pyrazolecarboxylates 63-66 with excellent regioselectivity (>97:3) using a one-pot procedure (Scheme 17) [91]. This method could be applied on $100 \mathrm{~g}$ scale without any problems related to exothermicity or stirring [92]. In the case of $\mathrm{N}$-substituted pyrazoles, esters $\mathbf{6 3 - 6 6}$ could be further functionalized by saponification, yielding carboxylic acids $\mathbf{6 7 - 7 0}$ as possible precursors for the synthesis of pyrazolecarboxamides towards SDHI ingredients (see Figure 1), and an additional decarboxylation step led to 3,5-bis(fluoroalkyl)pyrazoles 71-73 unsubstituted in position 4. On the other hand, the saponification conditions failed on NH-pyrazoles. Consequently, an alternative pathway was used to access to "naked" 3,5-bis(fluoroalkyl)-NH-pyrazole 71a via cleavage of the $\mathrm{N}-t \mathrm{Bu}$ moiety of $\mathrm{N}$ - $t \mathrm{Bu}-3,5$-bis(fluoroalkyl)pyrazoles in harsh acidic conditions prior to decarboxylation (Scheme 17) [91,92].

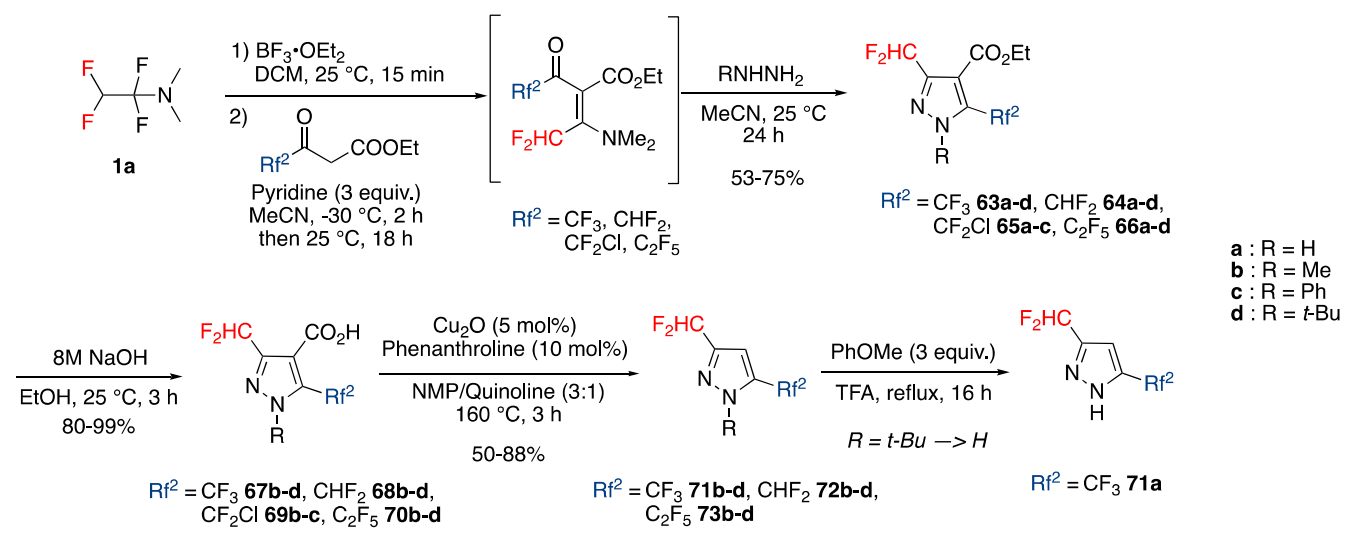

Scheme 17. First preparation of 3,5-bis(fluoroalkyl)pyrazolecarboxylates and -carboxylic acids and their decarboxylation to afford 3,5-bis(fluoroalkyl)-NH-pyrazoles. TFA, trifluoroacetic acid; NMP, $N$-methyl-2-pyrrolidone.

The strategy was also used by Leroux and coworkers for the synthesis of 3,5-bis(fluoroalkyl) isoxazolecarboxylates $\mathbf{7 4 - 7 7}$ by replacing hydrazines with hydroxylamine. The corresponding carboxylic acids $\mathbf{7 8 - 8 0}$ were also prepared similarly by hydrolysis, although the latter was carried out in acidic medium (Scheme 18).

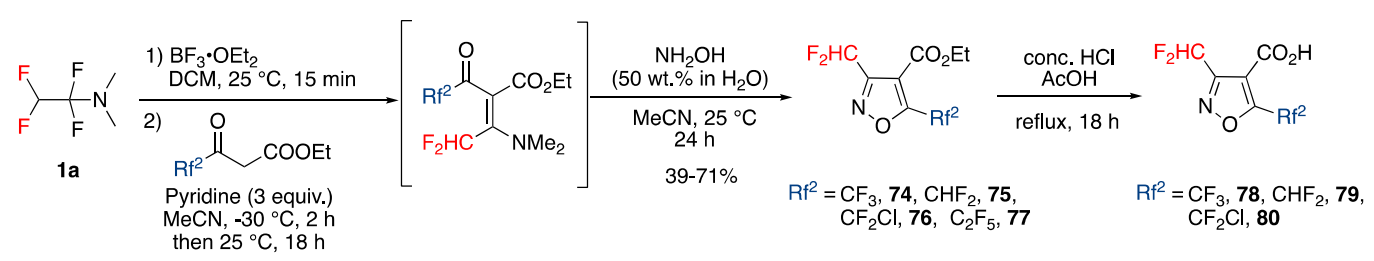

Scheme 18. First preparation of 3,5-bis(fluoroalkyl)isoxazolecarboxylates and carboxylic acids. 
Synthesis of 3,5-Bis(fluoroalkyl)-NH-pyrazoles from Azines

The method described above gave very good results in the access to 3,5-bis(fluoroalkyl)pyrazolecarboxylates 63-66. However, it suffered some limitations in the preparation of 3,5-bis(fluoroalkyl)-NH-pyrazoles 71a-harsh acidic conditions were needed to deprotect the $\mathrm{N}$-t $\mathrm{Bu}$ moiety. To circumvent this inconvenience and prepare efficiently unprecedented 3,5-bis(fluoro-alkyl)NH-pyrazoles 71a-j, another pathway was developed, based on the use of fluorinated azines 81a-e. The latter are a synthetic equivalent of fluorinated propanyl-2-ylidenehydrazines, whose free $\mathrm{NH}_{2}$ is revealed upon in situ hydrolysis of the benzophenone-derived imine subunit. By reaction with activated FARs $3 \mathbf{a}-\mathbf{c}$ followed by addition of acid, a cyclization would occur to provide the desired NH-pyrazoles (Scheme 19) [93].

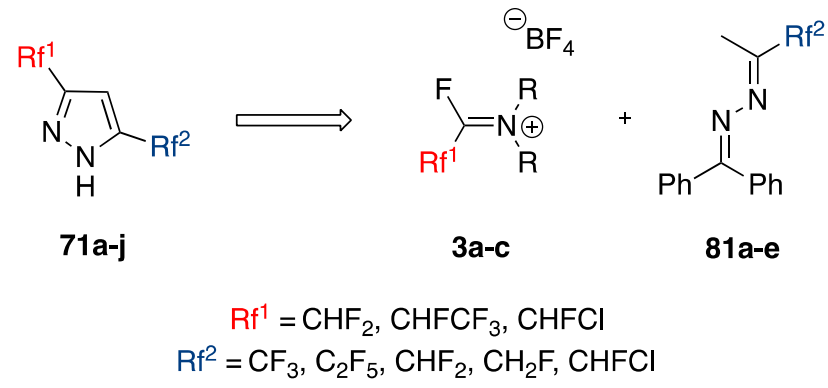

Scheme 19. The fluorinated azine-based strategy to access 3,5-bis(fluoroalkyl)-NH-pyrazoles.

The preparation of fluororinated azines 81a-e was straightforward. First benzophenone hydrazone 83 was prepared quantitatively by reaction of hydrazine hydrate with benzophenone 82 . Then fluoroacetones were condensed onto 83 to afford azines 81a-e with excellent yields (Scheme 20) [93].

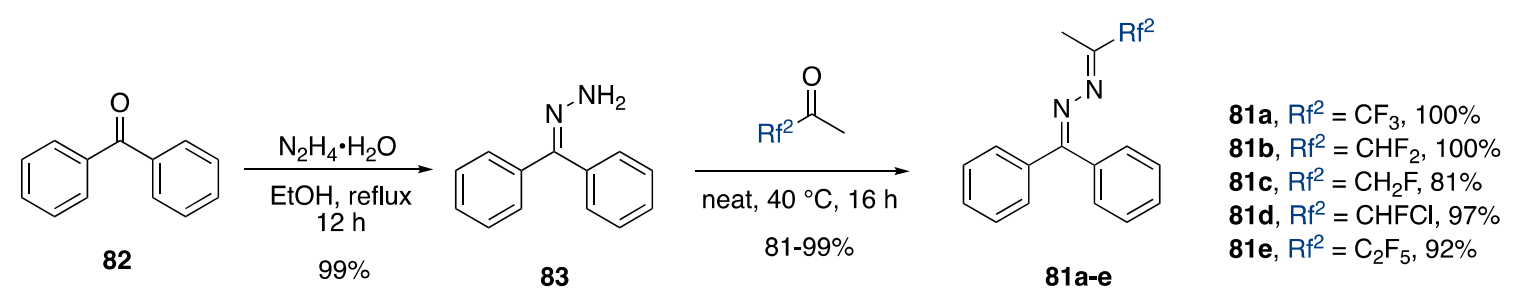

Scheme 20. Preparation of fluoroacetone-derived azines from benzophenone hydrazine.

Then, fluorinated azines 81a-e were reacted with activated FARs 3a-c (activation with $\mathrm{BF}_{3} \cdot \mathrm{OEt}_{2}$ ) to form vinamidinium intermediates $\mathbf{8 4 a - j}$. On the one hand, the latter led, upon hydrolysis by dilute aqueous $\mathrm{HCl}(1 \mathrm{~N})$, to $\beta$-(diphenylmethylenehydrazinyl)-bis(fluoroalkyl)-enones $\mathbf{8 5 a}$ - $\mathbf{h}$, which represent analogues of unsymmetrical fluorinated 1,3-diketones that are usually difficult to prepare. On the other hand, treatment of $\mathbf{8 4 a}-\mathbf{j}$ with concentrated $\mathrm{HCl}(12 \mathrm{~N})$ hydrolyzed the benzophenone imine moiety and triggered the ring-closing attack of the resulting hydrazine onto the electrophilic $\beta$-fluoro iminium. This step provided the desired 3,5-bis(fluoroalkyl)-NH-pyrazoles 71a-j with moderate to excellent yields (Scheme 21, pathway A). Interestingly, several of these pyrazoles could also be prepared from vinamides $\mathbf{8 5 a} \mathbf{a} \mathbf{h}$, by treating them with concentrated $\mathrm{HCl}$, to compare the reactivity of vinamides versus vinamidiniums. Whereas the cylization proceeded smoothly at room temperature from vinamidiniums $84 \mathbf{a}-\mathbf{j}$, heating the vinamides $85 a-h$ at $50{ }^{\circ} \mathrm{C}$ for $1-2 \mathrm{~h}$ was necessary to afford the cyclized products with lower yields (Scheme 21, pathway B). This difference in reactivity can be ascribed to the faster release of the secondary amine rather than that of water during the final aromatization step (Scheme 22) [93]. 


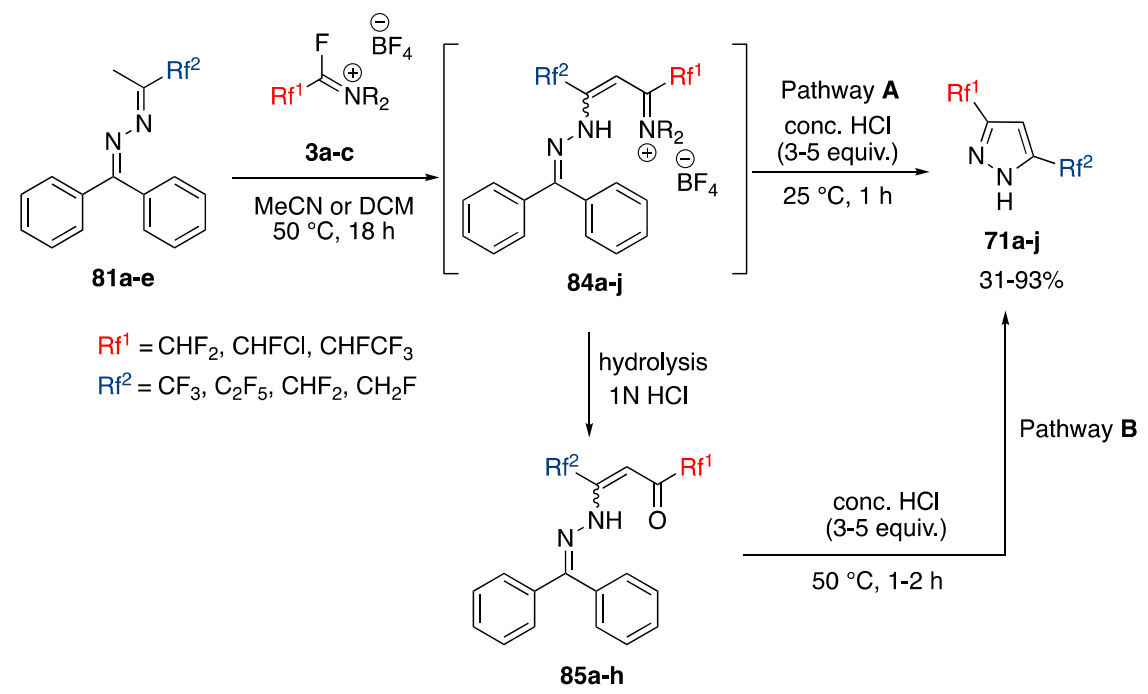

Scheme 21. Synthesis of novel 3,5-bis(fluoroalkyl)-NH-pyrazoles. Pathway A: from in situ formed vinamidiniums; Pathway B: from isolated vinamides.

Pathway $\mathbf{A}$ : from vinamidiums
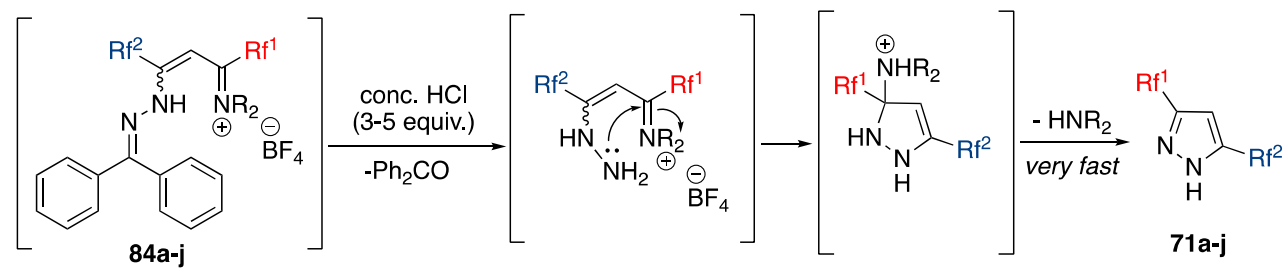

Pathway B : from vinamides<smiles>CCCCC(=O)/C=C(/[Ga])NN=C(c1ccccc1)c1ccccc1</smiles>
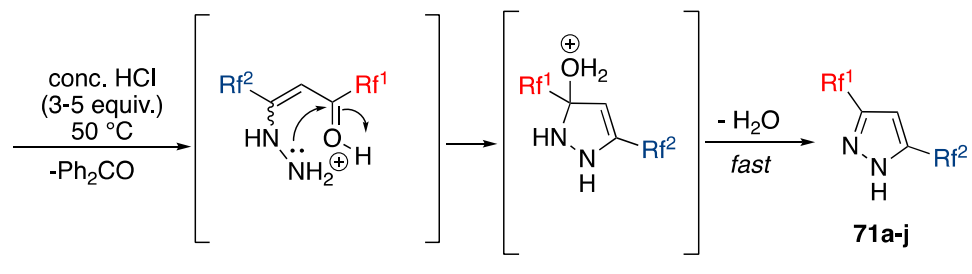

Scheme 22. Supposed mechanism for the intramolecular cyclization from vinamidinums. (Pathway A) or vinamides (Pathway B).

This strategy was also used to prepare 3-( $\left.\mathrm{CHF}_{2}\right)-5$-(fluoroaryl)-NH-pyrazoles $88 \mathbf{a}-\mathbf{d}$ from fluorinated acetophenones 86a-d with moderate yields (Scheme 23) [93].

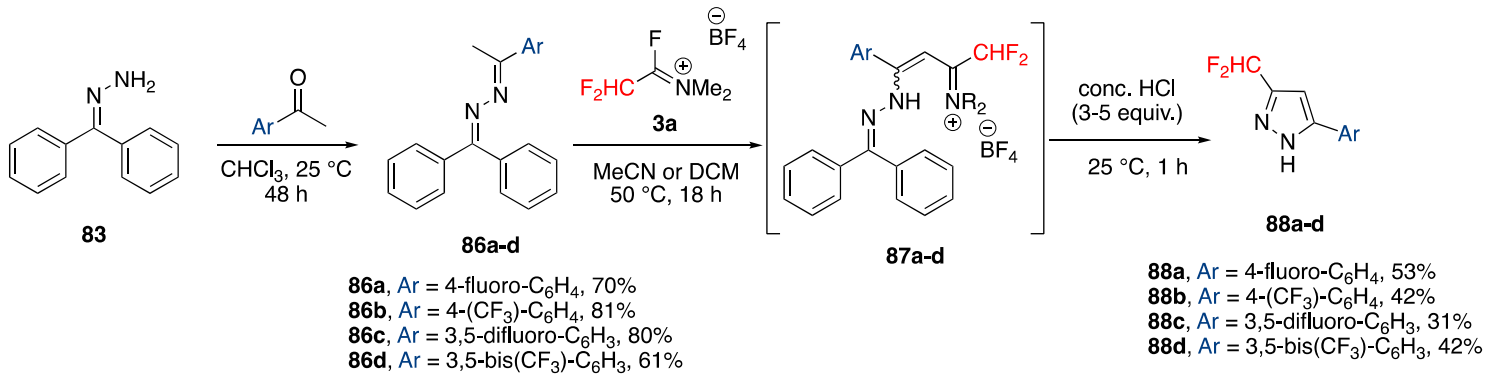

Scheme 23. Synthesis of unprecedented 3-( $\left.\mathrm{CHF}_{2}\right)-5-($ fluoroaryl)-NH-pyrazoles. 
This use of fluorinated azines represents the first efficient pathway to prepare unprecedented 3,5-bis(fluoroalkyl)-NH-pyrazoles. However, application of this method in industrial processes appears difficult, due to the tediousness of the complete removal of benzophenone released in the reaction. Moreover, the method was limited to the preparation of 3,5-bis(fluoroalkyl)-NH-pyrazoles. Several attempts of $\mathrm{N}$-methylation of these compounds were achieved and proved that the regioselective $\mathrm{N}$-functionalization is really difficult and mostly influenced by thermodynamic factors (unpublished results). Consequently, a new facile and efficient method was then reported to prepare series of 3,5-bis(fluoroalkyl)pyrazoles bearing not only a hydrogen or a methyl substituent, but also a large diversity of groups in position 1, while maintaining the control of regioselectivity [21]. This method will be described in the following sections.

Synthesis of 3,5-Bis(fluoroalkyl)-NH-pyrazoles from Ketimines

This new strategy was based on the addition of $N$-benzyl fluoroacetimines $89 a-c$ on activated FARs 3a-d. The reaction could be carried out under mild conditions $\left(25^{\circ} \mathrm{C}\right.$ in $\mathrm{MeCN}$ for up to $\left.1 \mathrm{~h}\right)$ to produce vinamidium intermediates $\mathbf{9 0 a}-\mathbf{j}$. These species can be directly reacted with hydrazine hydrate to afford 3,5-bis(fluoroalkyl)-NH-pyrazoles 71a-j under similarly mild conditions with moderate to excellent yields (Scheme 24). Interestingly, better results were attained with this ketimine-based method than with the azine-based route when starting from TFEDMA 1a. The trifluoromethoxy-subsituted FAR 1d, transferring a $\mathrm{CHFOCF}_{3}$ group, was also used and afforded new pyrazole scaffolds with very good yields (81-85\%). On the other hand, the Yarovenko and Ishikawa reagents proved overall less efficient (except when starting from the $\mathrm{CHF}_{2}$-ketimine) due once again to the lower reactivity of $\mathrm{N}, \mathrm{N}$-diethyl iminiums with regard to their dimethyl congeners, and to the lower purity of the starting commercial FARs $\mathbf{1 b}-\mathbf{c}$ [21].

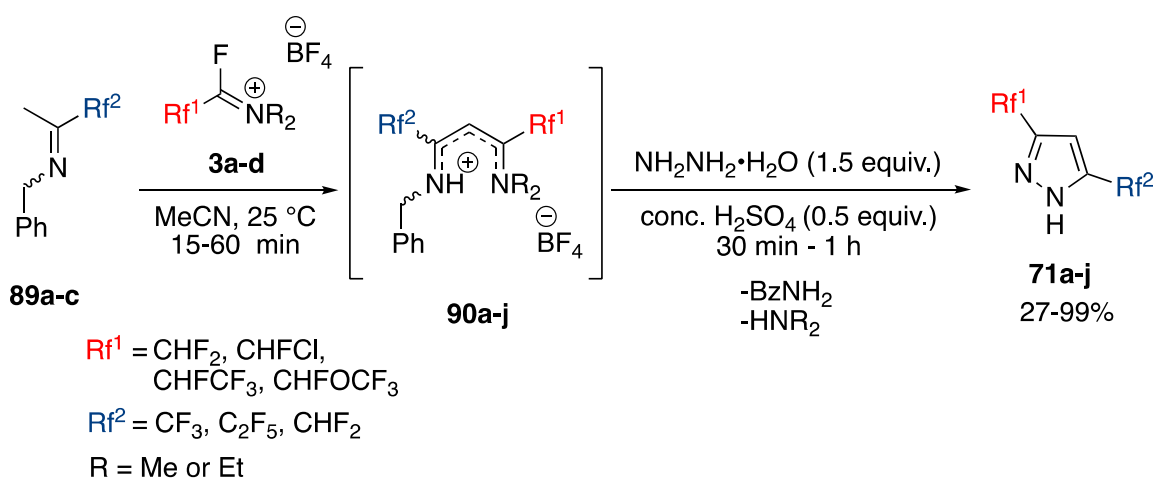

Scheme 24. Synthesis of 3,5-bis(fluoroalkyl)-NH-pyrazoles from fluorinated ketimines and hydrazine hydrate.

Synthesis of 3,5-Bis(fluoroalkyl)-NMe-pyrazoles from Ketimines

Unlike the synthesis of NH-pyrazoles from hydrazine hydrate, the access to NMe-pyrazoles implies an additional regioselectivity issue, due to the non-symmetrical nature of methyl hydrazine whose first nucleophilic attack can proceed via the $\mathrm{NH}_{2}$ or the NHMe groups (Scheme 25). The control of regioselectivity is critical since regioisomers $\mathbf{7 1}$ and $\mathbf{7 \mathbf { 1 } ^ { \prime }}$ are usually difficult to separate.

When vinamidinium intermediates $\mathbf{9 0 a}-\mathbf{j}$ were treated with methyl hydrazine under acidic conditions (Scheme 25, pathway A), the best results were again observed with TFEDMA 1a and the "OCF 3 -FAR" $1 \mathrm{~d}$, which led mainly to regioisomer $\mathbf{7 1}(\mathbf{7 1} / \mathbf{7 1}$ ' ratio $=71: 29$ to 100:0). For example, full regioselectivity in favour of isomer $\mathbf{7 1}$ was observed when $\mathbf{3 d}$ was opposed to $\mathrm{CF}_{3}$ - and $\mathrm{C}_{2} \mathrm{~F}_{5}$-ketimines. Conversely, the activated Yarovenko and Ishikawa reagents $\mathbf{3 b}-\mathbf{c}$ gave poorer results in terms of both reactivity and regioselectivity. Indeed, no reaction occurred with electron-poor and bulkier ketimines $\left(\mathrm{Rf}^{2}=\mathrm{CF}_{3}\right.$ and $\left.\mathrm{C}_{2} \mathrm{~F}_{5}\right)$; and while it proceeded with the $\mathrm{CHF}_{2}$-ketimine, a lower selectivity was observed, sometimes surprisingly in favour of isomer $\mathbf{7 1}^{\prime}$ [21]. 


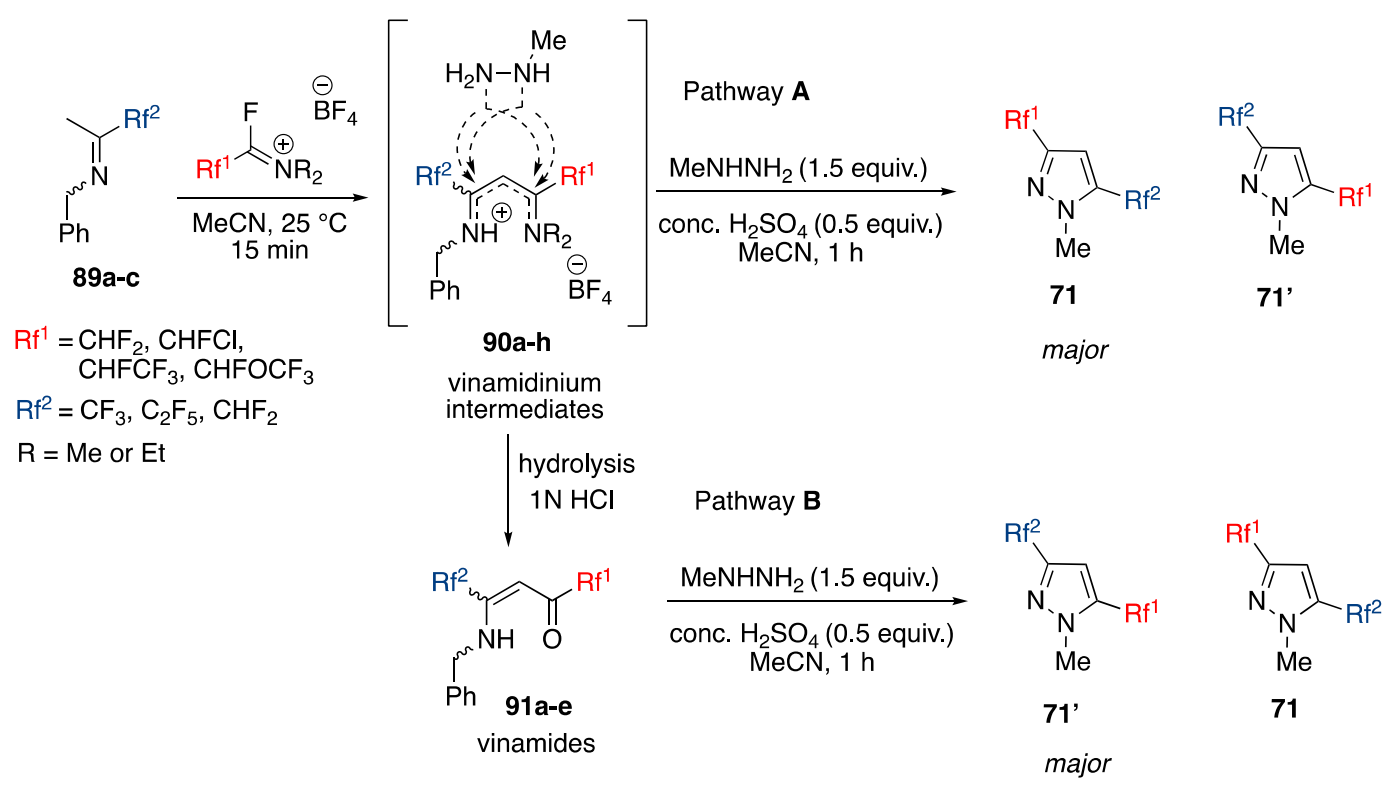

Scheme 25. Synthesis of 3,5-bis(fluoroaryl)-NMe-pyrazoles. Pathway A: from in situ formed vinamidiniums; Pathway B: from isolated vinamides.

To account for the regioselectivity, one can assume that in the case of TFEDMA and its $-\mathrm{OCF}_{3}$ analogue, which both lead to $\mathrm{N}, \mathrm{N}$-dimethyliminiums, the major isomer is formed due to two reasons. The first attack is believed to be more favorably affected by the $\mathrm{NH}_{2}$ moiety of methyl hydrazine, instead of the NHMe one, in order to avoid the steric clash between the methyl group and fluorinated substituents $\mathrm{Rf}^{1}$ or $\mathrm{Rf}^{2}$. Second, this first attack is driven by the release of the more volatile dimethylamine instead of benzylamine. On the other hand, for the two other FARs, the lower reactivity of more congested $\mathrm{N}, \mathrm{N}$-diethyliminium salts $3 \mathbf{b}-\mathbf{c}$ renders their attack by the $\mathrm{NH}_{2}$ group more difficult and affords mixed regioselectivities.

Vinamidiums 90a-j can also be hydrolysed to afford vinamides 91a-e, which can react afterwards with methyl hydrazine as 1,3-dielectrophiles (Scheme 25, pathway B). In this case, a reversed regioselectivity is observed with regard to the reaction of vinamidiniums. For example, treating unsymmetrical vinamide 4-(benzylamino)-1,1,5,5-tetrafluoropent-3-en-2-one $\left(\mathrm{Rf}^{1}=\mathrm{Rf}^{2}=\mathrm{CHF}_{2}\right)$ with methyl hydrazine led to isomer $\mathbf{7 1}^{\prime}$ as major product, presumably after initial addition of the $\mathrm{NH}_{2}$ moiety of methyl hydrazine onto the iminium tautomer which is more electrophilic than the carbonyl function of vinamides 91 [21].

Synthesis of 3,5-Bis(fluoroalkyl)-N-substituted-pyrazoles from Ketimines

After the development of efficient methods to prepare 3,5-bis(fluoroalkyl)-NH- and NMe-pyrazoles regioselectively, the synthesis of analogous pyrazoles bearing a wide diversity of substituents in position 1 was tackled, from commercially available substituted hydrazines. To avoid problems of regioselectivity, symmetrical bis $\left(\mathrm{CHF}_{2}\right)$ pyrazoles were first prepared, by means of either hydrazine hydrochloride salts in presence of $\mathrm{NEt}_{3}$ (helping to solubilize salts and the aromatization), or free hydrazines in presence of sulfuric acid. Vinamidinium intermediate 90a provided efficiently 1-alkyl- and 1-arylpyrazoles $\mathbf{9 2 a - d}$ with very good yields (90-99\%). For some hydrazines, especially the more hindered or more electron-deficient ones, microwave assistance was needed to afford the desired aryl pyrazoles $\mathbf{9 0 d}-\mathbf{f}$ with moderate yields (48-66\%). Some limitations were observed, such as the non-compatibility of the reaction conditions with acid-labile groups on the final pyrazoles (BOC, tosyl, $t \mathrm{Bu}$ and benzoyl under certain conditions) or a sluggish mixture in the case of $\mathbf{9 2 f}$, but various $\mathrm{N}$-substituted pyrazoles could still be obtained (Scheme 26) [21]. 


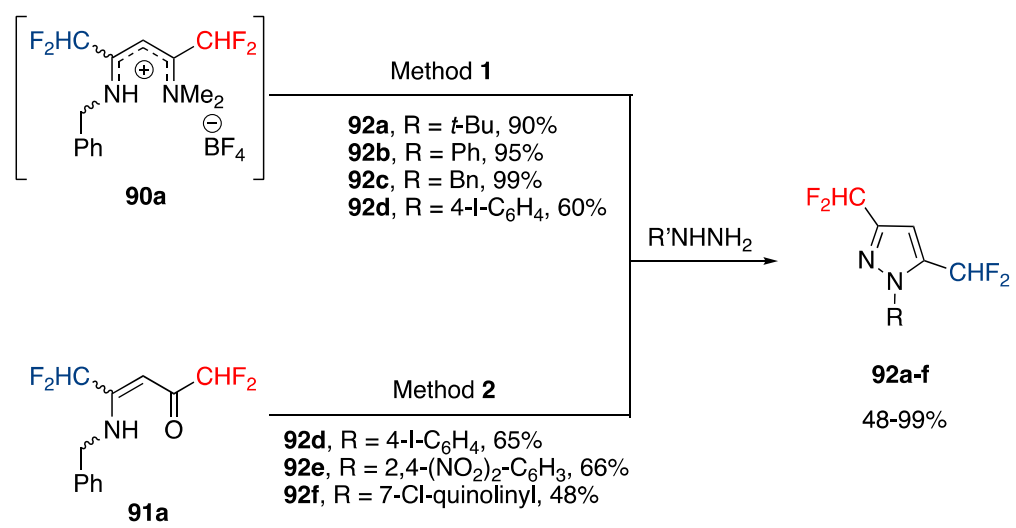

Scheme 26. Synthesis of various $\mathrm{N}$-substituted pyrazoles from vinamidiniums and vinamides. Method 1: hydrazine/conc. $\mathrm{H}_{2} \mathrm{SO}_{4}$, or hydrazine $\cdot \mathrm{HCl} / \mathrm{NEt}_{3}, \mathrm{MeCN}, 25-50{ }^{\circ} \mathrm{C}, 1 \mathrm{~h}$.; $\operatorname{Method} 2$ : hydrazine, conc. $\mathrm{H}_{2} \mathrm{SO}_{4}$, toluene/MeCN, $120-140{ }^{\circ} \mathrm{C}, \mathrm{MW}, 0.5-2 \mathrm{~h}$.

Interestingly, when 2,4-dinitrophenylhydrazine 93 was reacted with vinamidinium 90a, hydrazonamide 95 was formed in $83 \%$ yield, thus supporting the scenario where the first nucleophilic attack is effected by the $\mathrm{NH}_{2}$ end of the hydrazine onto the $\mathrm{N}, \mathrm{N}$-dimethyl iminium moiety of the vinamidinium (Scheme 27).

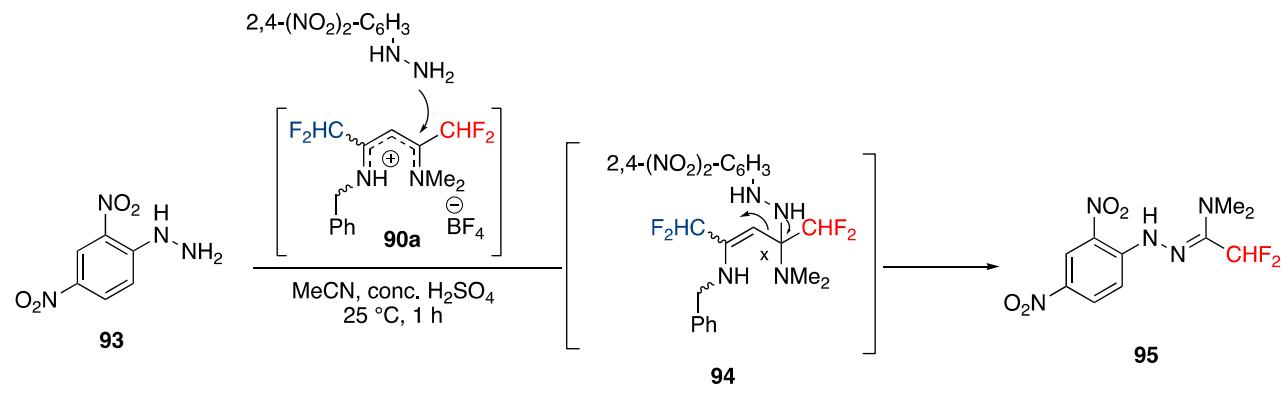

Scheme 27. Observed side-reaction product with 2,4-dinitrophenylhydrazine.

On the other hand, when hydrazines bearing a H-bonding N-substituent (benzoyl, BOC, carbamyl, 2-pyridinyl, tosyl), were used, the dehydration/deamination step (aromatization step) did not proceed and the corresponding hydroxy- or N-benzylaminopyrazolines were obtained (Scheme 28) [21]. As reported by several research groups, fluoroalkyl pyrazoles can be prepared from hydrazines and fluorinated 1,3-diketones or analogues, but the intermediate fluorinated 5-hydroxypyrazolines are often not dehydrated readily under the reaction conditions [94-96]. Since vinamidiniums 90 or vinamides 91 can be regarded as mono- or bis-iminium analogues of bis(fluoroalkyl)-1,3-diketones, it is not surprising that their reaction with hydrazines bearing a H-bonding N-substituent leads to non-aromatized products. Indeed, the latter substituent binds to the proton of the hydroxy or benzylamino group, thus increasing electron-density at $\mathrm{O}$ and $\mathrm{N}$ respectively, and therefore decreasing the acidity of the $\beta$-proton whose abstraction would lead to aromatization.

Several pyrazolines were thus isolated and demonstrated an excellent stability (Scheme 28) [21]. Interestingly, these experiments demonstrate the opposite reactivity of vinamidinium and vinamide intermediates. Indeed, 5-( $N$-benzylamino)pyrazolines $96 a-e$ were selectively prepared from bis $\left(\mathrm{CHF}_{2}\right.$ )-substituted vinamidinium 90a (Method 1) whereas 5-hydroxy-pyrazolines 97a-e were obtained from the corresponding vinamide 91a (Method 2). These results seem again to indicate that the first nucleophilic attack is carried out by the less hindered $\mathrm{NH}_{2}$ moiety of hydrazines onto the $\mathrm{N}, \mathrm{N}$-dimethyl iminium part of vinamidinium $\mathbf{9 0 a}$, while, in vinamide $91 \mathrm{a}$, this attack takes place on the $N$-benzyl iminium instead. 
Using the fluorinated polar protic solvent hexafluoropropan-2-ol (HFIP) involved a critical improvement in the reaction of vinamides (Method 3). This non-nucleophilic and highly H-bonding solvent proved highly appealing in the preparation of 5-hydroxypyrazolines $\mathbf{9 7 a - e}$ since it provided excellent yields in absence of strong Brønsted acid. This method was also used with non-symmetrical vinamides $\mathbf{9 1 b}-\mathbf{e}$ and for every $\mathrm{Rf}^{1} / \mathrm{Rf}^{2}$ couple, the reactivity of the $\mathrm{N}$-benzyl iminium moiety formed in situ was always higher than that of the fluoroalkyl ketone function towards attack by the $\mathrm{NH}_{2}$ end of the hydrazine. Four different unsymmetrical 5-hydroxy-pyrazolines $\mathbf{9 7 f}-\mathbf{i}$ were selectively formed with yield ranging from 62 to $99 \%$. Using a mixture of vinamides $\mathbf{9 1} \mathbf{d} / \mathbf{9 1} \mathbf{d} \mathbf{d}$ (65:35) provided respectively a mixture of 5-hydroxy-pyrazolines $\mathbf{9 7 h} / \mathbf{9 7 ^ { \prime }} \mathbf{h}$ (68:32) further separated by chromatography with almost complete conservation of the initial ratio (Scheme 28) [21].

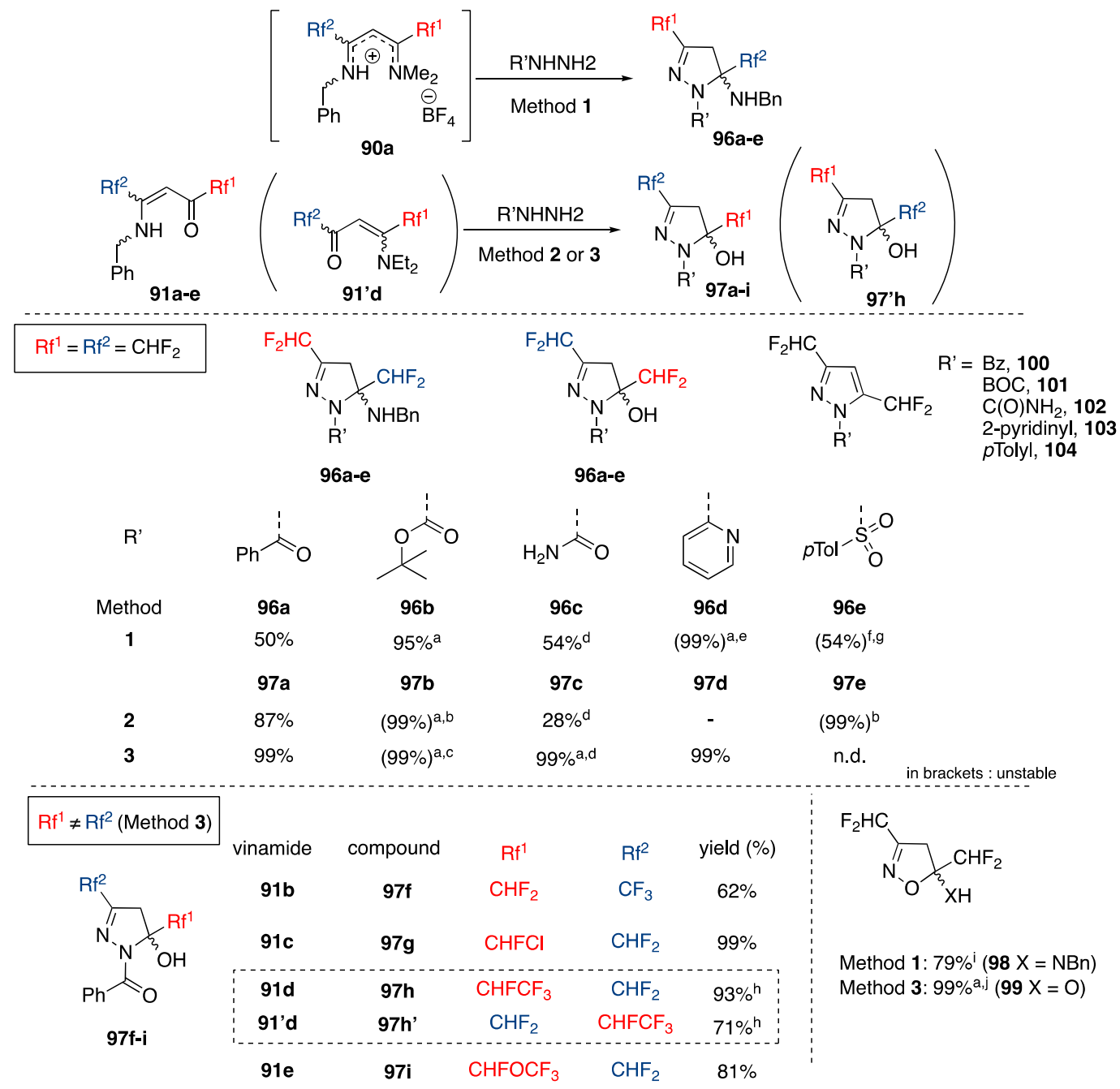

Scheme 28. Regioselective preparation of 5- $N$-benzylamino- and 5-hydroxypyrazolines and isoxazolines. Method 1: hydrazine, conc. $\mathrm{H}_{2} \mathrm{SO}_{4}, \mathrm{MeCN}, 25-50{ }^{\circ} \mathrm{C}, 1 \mathrm{~h}$. Method 2: hydrazine, toluene/MeCN, 120-140 ${ }^{\circ} \mathrm{C}$, MW, 0.5-2 h. Method 3: hydrazine, HFIP (hexafluoropropan-2-ol), 100-140 ${ }^{\circ} \mathrm{C}, 0.5-5 \mathrm{~h}$. ${ }^{\text {a }}{ }^{19} \mathrm{~F}$ NMR yield with $\mathrm{PhF}$ as internal standard. ${ }^{\mathrm{b}} \mathrm{R}$ group cleaved between $120{ }^{\circ} \mathrm{C}$ and $150{ }^{\circ} \mathrm{C}$. bis $\left(\mathrm{CHF}_{2}\right)$-NH-pyrazole 71 formed. ${ }^{\mathrm{c}} \mathrm{R}$ group cleaved between $80^{\circ} \mathrm{C}$ and $120^{\circ} \mathrm{C}$. bis $\left(\mathrm{CHF}_{2}\right)-\mathrm{NH}$-pyrazole 71 formed. ${ }^{d}$ prepared from a mixture of semicarbazide hydrochloride and $\mathrm{NEt}_{3}$, with no acid added. e Pyrazole 103 was isolated directly. ${ }^{\mathrm{f}} \mathrm{No}$ conc. $\mathrm{H}_{2} \mathrm{SO}_{4}$ used. ${ }^{\mathrm{g}} \mathrm{N}$-( $p$ Tolyl)-pyrazole (104) was separated by chromatography from pyrazoline $\mathbf{9 6 e}$ (29\% isolated). ${ }^{\mathrm{h}}$ Pyrazolines $\mathbf{9 7 h}$ and $\mathbf{9 7} \mathbf{h}$ were prepared from a 65/35 mixture of vinamides $91 \mathbf{d}$ and $\mathbf{9 1} \mathbf{d}$ and separated by chromatography. ${ }^{i}$ Hydroxylamine (50 wt. \% aq.) used instead of hydrazine. ${ }^{j}$ Hydroxylamine. $\mathrm{HCl}$ used instead hydrazine. 
The synthesis of the corresponding isoxazolines was achieved similarly using aqueous hydroxylamine or hydroxylamine hydrochloride instead of hydrazine. 5-(N-benzylamino)isoxazoline 98 and 5-hydroxypyrazoline 99 were isolated in very good yields (Scheme 28) [21]. This demonstrates that the more nucleophilic nitrogen attacks the more electrophilic iminium group in both starting vinamidinium salt 90a ( $N, N$-dimethyl iminium) and vinamide 91a ( $N$-benzyl iminium). The stabilization of the non-aromatized isoxazoline is permitted by either 1,4-H-bonding interactions or intermolecular H-bonding interactions.

Then, a selection of bis(fluoroalkyl)pyrazolines was successfully rearomatized under basic conditions (excess of pyridine) using thionyl chloride. N-benzoyl-5-hydroxypyrazoline 96 and $\mathrm{N}$-2-pyridinyl-5-hydroxypyrazoline 97 were readily and quantitatively dehydrated at room temperature to yield the corresponding pyrazoles 100 and 103. Conversely, reflux heating was required for the aromatization of $\mathrm{N}$-benzoyl-5-( $\mathrm{N}$-benzylamino)pyrazoline to provide pyrazole $\mathbf{1 0 0}$ and similarly for the $\mathrm{N}-(\mathrm{BOC})$-analogue, which afforded quantitatively the bis $\left(\mathrm{CHF}_{2}\right)-\mathrm{NH}$-pyrazole $\mathbf{7 1}$ due to the thermal instability of the BOC group (Scheme 29) [21].
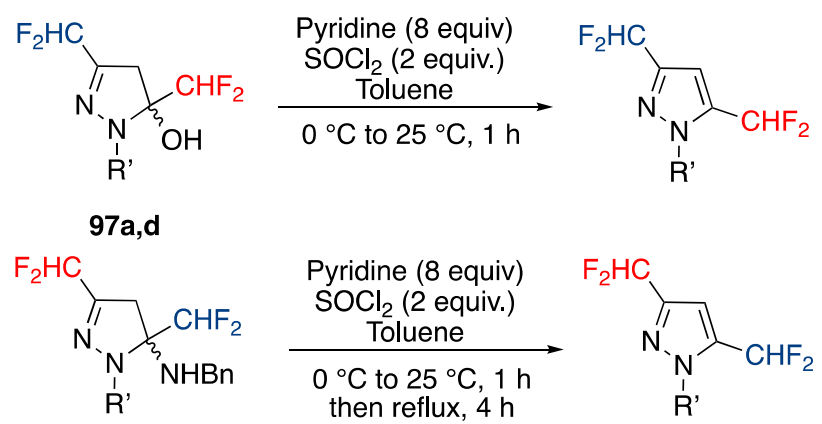

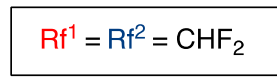

$\begin{array}{ccccc}\text { SM } & \mathrm{R} & 5-\mathrm{XH} & \text { Compound } & \text { yield } \\ \text { 97a } & \mathrm{C}(\mathrm{O}) \mathrm{Ph} & \mathrm{O} & 100 & 92 \%^{\mathrm{a}} \\ \text { 97d } & \text { 2-Pyridinyl } & \mathrm{O} & 103 & 99 \%^{\mathrm{a}} \\ \text { 96a } & \mathrm{C}(\mathrm{O}) \mathrm{Ph} & \mathrm{NBn} & 100 & 99 \%^{\mathrm{b}} \\ \text { 96b } & \mathrm{BOC} & \mathrm{NBn} & 101 & (99 \%)^{\mathrm{C}}\end{array}$

$96 a, b$

Scheme 29. Dehydration of several pyrazolines in basic conditions; ${ }^{a}$ Yield of isolated product. ${ }^{b}{ }^{19}$ F NMR yield with $\mathrm{PhF}$ as internal standard. ${ }^{\mathrm{c}}$ bis $\left(\mathrm{CHF}_{2}\right)-\mathrm{NH}$-pyrazole 73 formed after BOC (tert-butoxycarbonyle) cleavage.

To complete the investigation, a variety of functional groups (halogen, nitro, amine, aldehyde, carboxylic acid, boronate) was introduced into the 4-position of the model substrate, 3,5-bis $\left(\mathrm{CHF}_{2}\right)$ NH-pyrazole 71, to improve the applicability of 3,5-bis(fluoroalkyl)pyrazoles [21].

\subsubsection{Synthesis of 2,4-Bis(fluoroalkyl)-substituted Quinoline Derivatives}

The previous section covered the reaction of FARs with fluorinated $N$-benzylketimines to prepare 3,5-bis(fluoroalkyl)pyrazoles. When $N$-aryl fluoroketimines are used instead, the reaction outcome drastically changes. In this case, the vinamidinium intermediate readily cyclizes without addition of a hydrazine or of hydroxylamine as cyclization partner. The highly electrophilic distal fluorinated iminium indeed undergoes attack by the aryl substituent of the remote nitrogen, in a Friedel-Crafts-type reaction, to finally afford 2,4-bis(fluoroalkyl)quinolines after rearomatization. The synthesis of quinoline derivatives bearing two fluorinated groups in both positions 2 and 4 is scarcely described; only syntheses of bis(trifluoromethylated)quinolines were reported [97-100]. The use of FARs allowed to prepare in one step, from two series of variously substituted aryl fluoroketimines 106a-1 and 107a-u, a large diversity of 2,4-bis(fluoroalkyl)quinolines $109 a-1$ and $\mathbf{1 1 0 u}$ bearing different fluorinated groups on the pyrido moiety and various substituents on the benzo ring under mild conditions. Interestingly, complete regioselectivity was always observed, obviously with $N$-(4-substituted-phenyl)imines, but also with the 2- and 3- substituted analogues. The reaction yields were dependent on the nature of the substituents $\left(\mathrm{R}^{1}\right)$, of the starting aniline of the $\mathrm{Rf}^{1}$ and $\mathrm{Rf}^{2}$ groups and the $\mathrm{R}$ substituents of the FAR 
nitrogen atom. Indeed, the critical intermediate 108, where the nucleophilic and electrophilic termini required for cyclization are part of the same molecule and heavily conjugated, is strongly affected by the electronic and steric effects of all substituents decorating the $N$-aryl vinamidinium backbone (Scheme 30) [101].

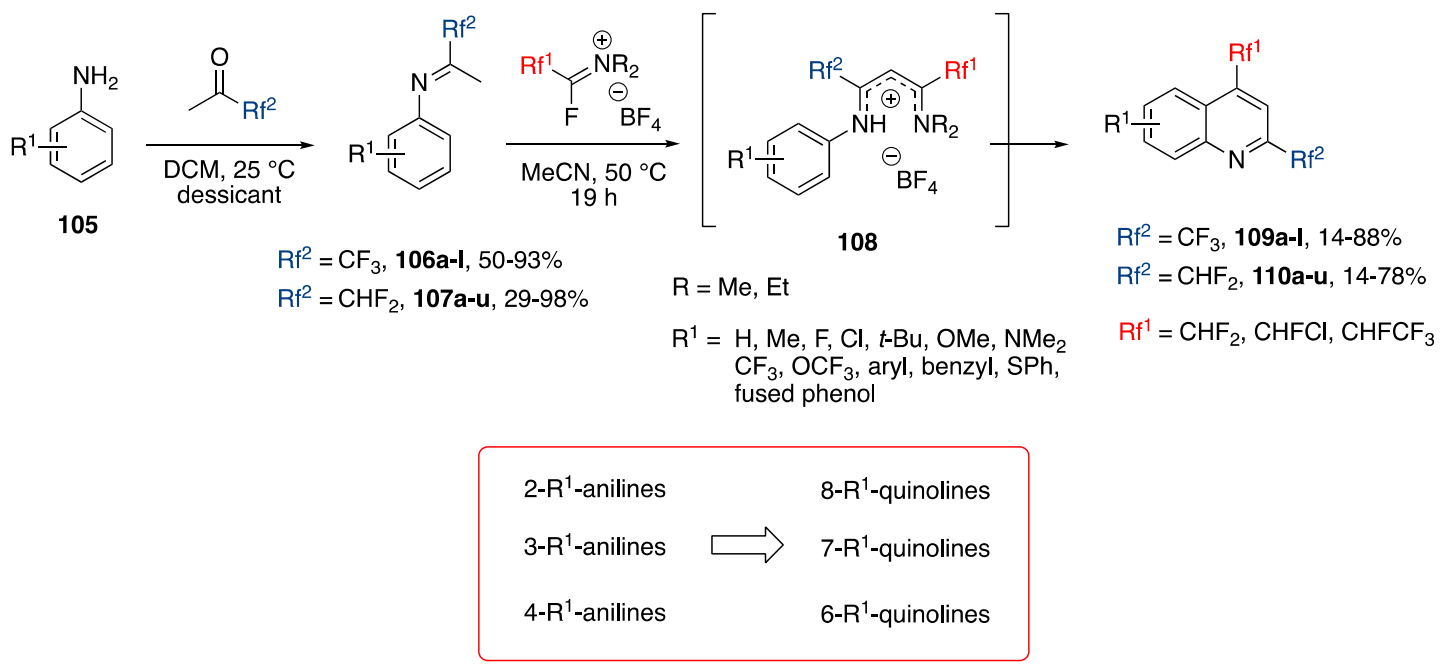

Scheme 30. Preparation of 2,4-bis(fluoroalkyl)quinolines from aryl fluoroketimines.

\subsection{Reaction with Allylic and Propargylic Alcohols}

In previous Sections 3.2-3.4 we have described the uses of FARs to perform the dehydroxy-fluorination of alcohols, with no carbon of the FAR present in the final product, and reactions where all carbons of the FAR are present in the product but only one, the carbon of the iminium, undergoes transformation.

When allylic or propargylic alcohols are reacted with FARs, another, distinct outcome is revealed, with two carbons of the FAR being transformed and incorporated in the reaction product. Indeed, the reaction between the Ishikawa reagent 1c and the hydroxyl function of allylic 111 and 116 or propargylic 120 alcohols affords iminium intermediates 112, 117 and 121. Due to the acidic proton in $\alpha$ position of the imidate carbon, the latter undergoes tautomery leading to the enamine form which can then react intramolecularly as a nucleophile to form different fluoralkylated molecules. Thus, $\alpha$-fluoro- $\alpha$-trifluoromethyl- $\gamma$-lactones 115 can be formed stereospecifically from Ishikawa's reagent and racemic or enantioenriched $\gamma$-hydroxy- $\alpha, \beta$-unsaturated sulfones 111 (Scheme 31, pathway A) [102]. The diastereoselective formation of 2-fluoro-2-trifluoromethyl-4-alkenamides 119 was also reported from 1c and (Z)-allylic alcohols 116 via a Claisen rearrangement (Scheme 31, pathway B) [103]. The same technique was reproduced from propargyl alcohols $\mathbf{1 2 0}$ to afford the related allenes 123 with good yields (Scheme 31, pathway C) [104]. These reactions were then applied to the diastereoselective and enantioselective synthesis of $\alpha$-trifluoromethylated $\alpha$-amino acid derivatives from $\gamma$-hydroxy- $\alpha$-fluoro- $\alpha$-trifluoromethyl carboxamides [105]. In the end, although this reactivity mode of FARs has only been reported for the Ishikawa reagent, one can assume that other FARs can be compatible.

\subsection{Transformation of the Three Carbons of the Ishikawa Reagent}

Finally, another application of FARs makes a constructive use of all carbons of the FAR, which are al transformed and incorporated in the reaction product. The Ishikawa reagent, like other FARs, can be easily hydrolysed to form the corresponding acetamide $4 c$, which can then be treated with a polar organometallic species (ArMgX) to afford acylated products, as detailed in Section 3.3. The $\alpha$ position of this ketone is relatively acidic and can be deprotonated by an alkoxide, to form in situ 
the corresponding difluoromethylene upon elimination of fluoride. The transient $\beta$, $\beta$-difluoroenone then reacts quickly with excess alkoxide to afford $\alpha$-fluoro- $\beta$-ketoesters $126 \mathbf{a}-\mathrm{g}$. It is important to note that this step is possible only when starting from the Ishikawa reagent, which is the only FAR among $\mathbf{1 a}-\mathbf{d}$ to be derived from a 3-carbon alkene. The resulting $\alpha$-fluoro- $\beta$-ketoesters 126a-g possess two electrophilic sites and can react with dinucleophiles to provide fluorinated heterocycles. For example, monofluorinated pyrazoles and coumarins can be prepared by reaction with hydrazine and phenols respectively (Scheme 32) [106].

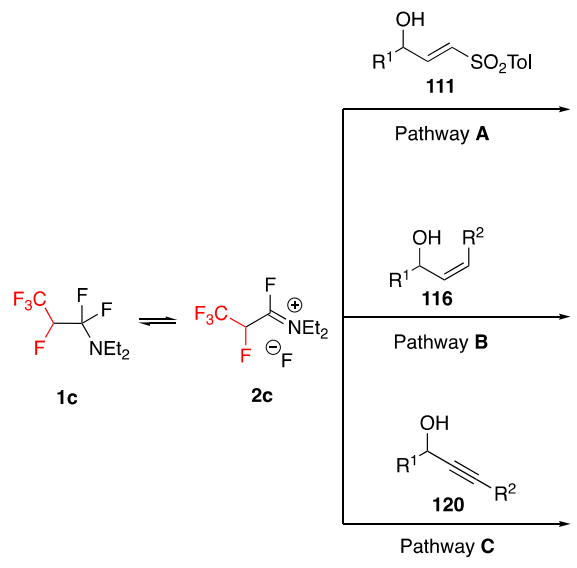

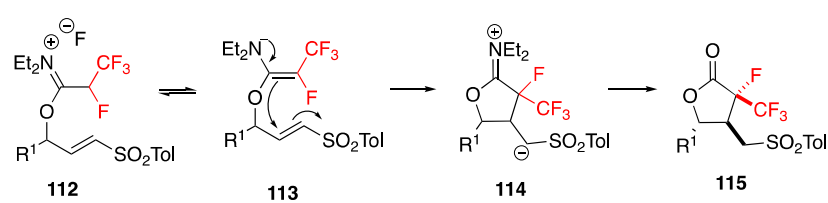

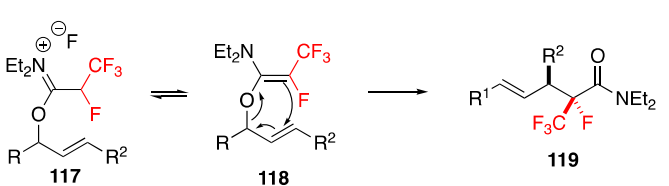<smiles>[R]C#CC([R])OC(=[N+]([O-])CC)C(F)(F)F</smiles>

12

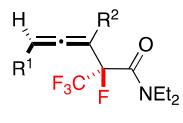

123

Scheme 31. Reaction between the Ishikawa reagent and allylic or propargylic alcohols.

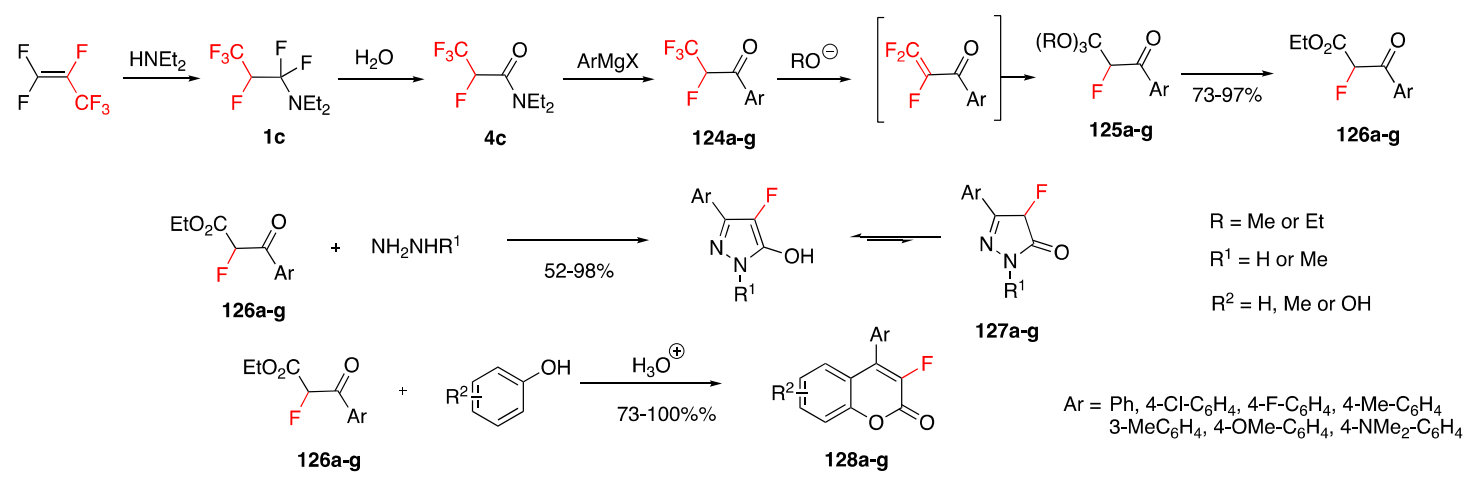

Scheme 32. Synthesis of fluorinated heterocycles from the hydrolyzed Ishikawa reagent [106].

\section{Conclusions}

While fluoroalkyl amino reagents were discovered more than a half century ago, their utilization was really diversified in 1975 when Wakselman et al. published their first applications as fluoroacylating agents for aromatics. The chemistry of FARs underwent a second impulse at the beginning of the 21st century when the need for fluorinated heterocycle-based crop protection ingredients by agrochemical companies focused on difluoromethylpyrazoles. Indeed, $3-\mathrm{CHF}_{2}$-pyrazolecarboxamide derivatives showed high activity as SDHI fungicides and several analogues were marketed by agro companies. In order to enhance the diversity and activities of these active ingredients, novel structures were sought and their preparation was studied. The development of new methods to introduce diverse emergent fluorinated substituents on heterocycles was necessary and FARs showed very interesting applications. Numerous fluorinated N-based 5- and 6-membered heterocycles bearing "classical" or new fluorinated substituents, particularly $\mathrm{CF}_{3}, \mathrm{C}_{2} \mathrm{~F}_{5}, \mathrm{CHF}_{2}, \mathrm{CHFCl}_{\text {, }}$ $\mathrm{CHFCF}_{3}$ or $\mathrm{CHFOCF}_{3}$ were successfully prepared using fast, efficient, robust and scalable methods. 
Acknowledgments: We thank the CNRS France (Centre National de la Recherche Scientifique), the University of Strasbourg and are very much grateful to Bayer S.A.S. for a grant to E.S., F.A. and B.C. The French Fluorine Network (GIS CNRS Fluor) is also acknowledged.

Conflicts of Interest: The authors declare no conflict of interest.

\section{References}

1. Bégué, J.-P.; Bonnet-Delpon, D. Fluorinated Drugs. In Bioorganic and Medicinal Chemistry of Fluorine; John Wiley \& Sons, Inc.: Hoboken, NJ, USA, 2008; pp. 279-351.

2. Jeschke, P. The unique role of fluorine in the design of active ingredients for modern crop protection. ChemBioChem 2004, 5, 571-589. [CrossRef] [PubMed]

3. Leroux, F.; Jeschke, P.; Schlosser, M. $\alpha$-Fluorinated Ethers, Thioethers, and Amines: Anomerically Biased Species. Chem. Rev. 2005, 105, 827-856. [CrossRef] [PubMed]

4. Hong, W. Agricultural Products Based on Fluorinated Heterocyclic Compounds. In Fluorinated Heterocyclic Compounds; John Wiley \& Sons, Inc.: Hoboken, NJ, USA, 2009; pp. 397-418.

5. Cartwright, D. Recent Developments in Fluorine-Containing Agrochemicals. In Organofluorine Chemistry: Principles and Commercial Applications; Banks, R.E., Smart, B.E., Tatlow, J.C., Eds.; Springer: Boston, MA, USA, 1994; pp. 237-262.

6. Fujiwara, T.; O'Hagan, D. Successful fluorine-containing herbicide agrochemicals. J. Fluorine Chem. 2014, 167, 16-29. [CrossRef]

7. Theodoridis, G. Fluorine-Containing Agrochemicals: An Overview of Recent Developments. In Advances in Fluorine Science; Alain, T., Ed.; Elsevier: Amsterdam, The Netherlands, 2006; Volume 2, pp. 121-175.

8. Jeschke, P. The unique role of halogen substituents in the design of modern agrochemicals. Pest Manag. Sci. 2010, 66, 10-27. [CrossRef] [PubMed]

9. Muller, K.; Faeh, C.; Diederich, F. Fluorine in pharmaceuticals: Looking beyond intuition. Science 2007, 317, 1881-1886. [CrossRef] [PubMed]

10. Purser, S.; Moore, P.R.; Swallow, S.; Gouverneur, V. Fluorine in medicinal chemistry. Chem. Soc. Rev. 2008, 37, 320-330. [CrossRef] [PubMed]

11. O'Hagan, D. Fluorine in health care: Organofluorine containing blockbuster drugs. J. Fluorine Chem. 2010, 131, 1071-1081. [CrossRef]

12. Landelle, G.; Panossian, A.; Leroux, F.R. Trifluoromethyl ethers and -thioethers as tools for medicinal chemistry and drug discovery. Curr. Top. Med. Chem. 2014, 14, 941-951. [CrossRef] [PubMed]

13. Zhou, Y.; Wang, J.; Gu, Z.; Wang, S.; Zhu, W.; Acena, J.L.; Soloshonok, V.A.; Izawa, K.; Liu, H. Next Generation of Fluorine-Containing Pharmaceuticals, Compounds Currently in Phase II-III Clinical Trials of Major Pharmaceutical Companies: New Structural Trends and Therapeutic Areas. Chem. Rev. 2016, 116, 422-518. [CrossRef] [PubMed]

14. Pruett, R.L.; Barr, J.T.; Rapp, K.E.; Bahner, C.T.; Gibson, J.D.; Lafferty, R.H. Reactions of Polyfluoro Olefins. II. Reactions with Primary and Secondary Amines. J. Am. Chem. Soc. 1950, 72, 3646-3650. [CrossRef]

15. Knunyants, I.L.; German, L.S.; Dyatkin, B.L. Reactions of fluoro olefins Communication 6. Reactions of perfluoroisobutylene and perfluoropropene with nucleophilic reagents. Russ. Chem. Bull. 1956, 5, 1387-1394. [CrossRef]

16. Yarovenko, N.N.; Raksha, M.A. Fluorination with $\alpha$-fluorinated amines. Zh. Obshch. Khim. 1959, 29, $2159-2163$.

17. England, D.C.; Melby, L.R.; Dietrich, M.A.; Lindsey, R.V. Nucleophilic Reactions of Fluoroölefins. J. Am. Chem. Soc. 1960, 82, 5116-5122. [CrossRef]

18. Takaoka, A.; Iwakiri, H.; Ishikawa, N. F-Propene-Dialkylamine Reaction-Products as Fluorinating Agents. Bull. Chem. Soc. Jpn. 1979, 52, 3377-3380. [CrossRef]

19. Petrov, V.A.; Swearingen, S.; Hong, W.; Chris Petersen, W. 1,1,2,2-Tetrafluoroethyl-N,N-dimethylamine: A new selective fluorinating agent. J. Fluorine Chem. 2001, 109, 25-31. [CrossRef]

20. Walkowiak, J.; Koroniak, H. Preparation of $\alpha$-Fluoro Amino and $\alpha$-Fluoro Enamino Reagents. In Efficient Preparations of Fluorine Compounds; John Wiley \& Sons, Inc.: Hoboken, NJ, USA, 2012; pp. 379-384.

21. Schmitt, E.; Panossian, A.; Vors, J.P.; Funke, C.; Lui, N.; Pazenok, S.; Leroux, F.R. A Major Advance in the Synthesis of Fluoroalkyl Pyrazoles: Tuneable Regioselectivity and Broad Substitution Patterns. Chem. Eur. J. 2016, 22, 11239-11244. [CrossRef] [PubMed] 
22. Pazenok, S.; Vors, J.-P.; Leroux, F.R.; Schmitt, E. Process for Preparing Substituted Pyrazoles Containing Haloalkoxy-and Haloalkylthio Groups from $\alpha, \alpha$-Dihaloalkylamines and Ketimines. WO2016207167, 21 June 2016.

23. Viehe, H.G.; Janousek, Z. The Chemistry of Dichloromethylenammonium Salts ("Phosgenimonium Salts"). Angew. Chem. Int. Ed. Engl. 1973, 12, 806-818. [CrossRef]

24. Ishikawa, N.; Kitazume, T.; Takaoka, A. Fluorinating Agents for O-Functional Groups. J. Synth. Org. Chem. Jpn. 1979, 37, 606-611. [CrossRef]

25. Hamman, S.; Barrelle, M.; Tetaz, F.; Beguin, C.G. Acide fluoro-2 phenyl-2 acetique: Synthesis, configuration absolute et emploi comme agent chiral de derivation. J. Fluorine Chem. 1987, 37, 85-94. [CrossRef]

26. Spero, G.B.; Pike, J.E.; Lincoln, F.H.; Thompson, J.L. A new approach to $16 \alpha$-halo corticoids. II The synthesis of $16 \alpha$-fluoro and $16 \alpha$-chloro corticoids. Steroids 1968, 11, 769-786. [CrossRef]

27. Knox, L.H.; Velarde, E.; Berger, S.; Cuadriello, D.; Cross, A.D. Steroids. CCXL. The Reaction of Steroidal Alcohols with 2-Chloro-1,1,2-trifluorotriethylamine. J. Org. Chem. 1964, 29, 2187-2195. [CrossRef]

28. Allen, G.R.; Weiss, M.J. New Progestational Agents. Nonclassical 17-Alkylpregnene Structures. J. Med. Chem. 1964, 7, 684-686. [CrossRef] [PubMed]

29. Ayer, D.E. The Synthesis of 15ß-Fluoro Corticoids. J. Med. Chem. 1963, 6, 608-610. [CrossRef] [PubMed]

30. Ayer, D.E. A new method for the preparation of fluoro steroids. Tetrahedron Lett. 1962, 3, $1065-1069$. [CrossRef]

31. Knox, L.H.; Velarde, E.; Berger, S.; Cuadriello, D.; Cross, A.D. The Reactions of Steroidal Alcohols with 2-chloro-1,1,2-trifluorotriethylamine. Tetrahedron Lett. 1962, 3, 1249-1255. [CrossRef]

32. Crabbe, P.; Carpio, H.; Velarde, E.; Fried, J.H. Chemistry of difluorocyclopropenes. Application to the synthesis of steroidal allenes. J. Org. Chem. 1973, 38, 1478-1483. [CrossRef]

33. Knox, L.H.; Velarde, E.; Berger, S.; Delfín, I.; Grezemkovsky, R.; Cross, A.D. Steroids. CCLXXXIV. Reactions of 19-Hydroxy- $\Delta^{5}$-3-acetoxy Steroids with Diethyl(2-chloro-1,1,2-trifluoroethyl)amine. J. Org. Chem. 1965, 30, 4160-4165. [CrossRef] [PubMed]

34. Wood, K.R.; Fisher, D.; Kent, P.W. Fluorocarbohydrates. Part XIV. Reaction of N-(2-chloro-1,1,2-trifluoroethyl) diethylamine with some $O$-isopropylidene sugars. J. Chem. Soc. 1966, 21, 1994-1997. [CrossRef]

35. Bergmann, E.D.; Cohen, A.M. Organic Fluorine Compounds. Part 43. Applications of (1,1,2-Trifluoro2-Chloroethyl)-Diethylamine as Fluorinating Agent. Isr. J. Chem. 1970, 8, 925-933. [CrossRef]

36. Bateson, J.H.; Cross, B.E. Reactions of 2-chloro-NN-diethyl-1,1,2-trifluoroethylamine with alcohols. Part I. Preparation of $2 \beta$ - and $4 \beta$-fluorogibberellins. J. Chem. Soc., Perkin Trans. 1 1974, 2409-2413. [CrossRef]

37. Müller, B.; Peter, H.; Schneider, P.; Bickel, H. New $\beta$-Lactam-Antibiotics. Fluorinated Cephalosporins. Preliminary Communication. Modifikationen von Antibiotika. 15. Mitteilung. Helv. Chim. Acta 1975, 58, 2469-2473. [CrossRef] [PubMed]

38. O'Hagan, D. Preparation of monofluorocarboxylic acids using $N, N$-diethyl-1,1.2,3,3,3-hexafluoropropylamine. J. Fluorine Chem. 1989, 43, 371-377. [CrossRef]

39. Watanabe, S.; Fujita, T.; Usui, Y.; Kitazume, T. Fluorination of hydroxyesters with N,N-diethyl-1,1,2,3,3,3hexafluoropropylamine. J. Fluorine Chem. 1986, 31, 247-253. [CrossRef]

40. Watanabe, S.; Fujita, T.; Sakamoto, M.; Kuramochi, T.; Kitazume, T. Reactions of monoesters of ethylene glycol with $N, N$-diethyl-1,1,2,3,3,3-hexafluoropropylamine. J. Fluorine Chem. 1987, 36, 361-372. [CrossRef]

41. Watanabe, S.; Fujita, T.; Sakamoto, M.; Endo, H.; Kitazume, T. Fluorination of aromatic $\alpha$-hydroxyesters with N,N-diethyl-1,1,2,3,3,3-hexafluoropropaneamine. J. Fluorine Chem. 1990, 47, 187-192. [CrossRef]

42. Bresciani, S.; Slawin, A.M. Z.; O'Hagan, D. A regio- and stereoisomeric study of allylic alcohol fluorination with a range of reagents. J. Fluorine Chem. 2009, 130, 537-543. [CrossRef]

43. Araki, K.; Katagiri, T.; Inoue, M. Facile synthesis of 1,7,8-trifluoro-2-naphthol via DMAP catalyzed cycloaromatization. J. Fluorine Chem. 2014, 157, 41-47. [CrossRef]

44. Tan, X.; Soualmia, F.; Furio, L.; Renard, J.-F.; Kempen, I.; Qin, L.; Pagano, M.; Pirotte, B.; El Amri, C.; Hovnanian, A.; et al. Toward the First Class of Suicide Inhibitors of Kallikreins Involved in Skin Diseases. J. Med. Chem. 2015, 58, 598-612. [CrossRef] [PubMed]

45. Wang, Z.H.; Zheng, C.; Li, F.; Zhao, L.; Chen, F.E.; He, Q.Q. An Efficient Enantioselective Synthesis of Florfenicol Based on Sharpless Asymmetric Dihydroxylation. Synthesis 2012, 44, 699-704. 
46. Ando, T.; Koseki, N.; Yasuhara, I.; Matsuo, N.; Ishiwatari, T. Synthesis of Fluorinated Pyrethroids: Conversion of Pyrethroid Metabolites into Some Insecticidal Fluorinated Derivatives. Biosci. Biotechnol. Biochem. 1992, 56, 1581-1583. [CrossRef]

47. Tanaka, M.; Moriguchi, T.; Kizuka, M.; Ono, Y.; Miyakoshi, S.I.; Ogita, T. Microbial hydroxylation of zofimarin, a sordarin-related antibiotic. J. Antibiot. 2002, 55, 437-441. [CrossRef] [PubMed]

48. Schumacher, D.P.; Clark, J.E.; Murphy, B.L.; Fischer, P.A. An efficient synthesis of florfenicol. J. Org. Chem. 1990, 55, 5291-5294. [CrossRef]

49. Cantrell, G.L.; Filler, R. Further studies on the synthesis of $\alpha$-fluoro carbonyl compounds. J. Fluorine Chem. 1985, 27, 35-45. [CrossRef]

50. Hudlicky, M.; Merola, J.S. New stereospecific syntheses and X-ray diffraction structures of (-)-D-erythroand (+)-L-threo-4-fluoroglutamic acid. Tetrahedron Lett. 1990, 31, 7403-7406. [CrossRef]

51. Hudlický, M. Stereospecific syntheses of all four stereoisomers of 4-fluoroglutamic acid. J. Fluorine Chem. 1993, 60, 193-210. [CrossRef]

52. Gerus, I.I.; Mironets, R.V.; Shaitanova, E.N.; Kukhar, V.P. Synthesis of new $\beta$-trifluoromethyl containing GABA and $\beta$-fluoromethyl containing N-benzylpyrrolidinones. J. Fluorine Chem. 2010, 131, 224-228. [CrossRef]

53. Kitamoto, T.; Ozawa, T.; Abe, M.; Marubayashi, S.; Yamazaki, T. Incorporation of fluoroprolines to proctolin: Study on the effect of a fluorine atom toward peptidic conformation. J. Fluorine Chem. 2008, 129, $286-293$. [CrossRef]

54. Takahashi, Y.; Ogawa, T. Total synthesis of cyclomaltohexaose. Carbohydr. Res. 1987, 164, 277-296. [CrossRef]

55. Van der Steen, R.; Groesbeek, M.; van Amsterdam, L.J.P.; Lugtenburg, J.; van Oostrum, J.; de Grip, W.J. All E-10,20-methanoretinoylopsin, light-stable rhodopsin. Synthesis and spectroscopy of all E-10,20-methanoand all-E-retinoyl fluoride and their reaction with bovine opsin. Recl. Trav. Chim. Pays-Bas 1989, 108, 20-27. [CrossRef]

56. Hansen, P.E.; Nicolaisen, F.M.; Schaumburg, K. Deuterium isotope effects on nuclear shielding. Directional effects and nonadditivity in acyl derivatives. J. Am. Chem. Soc. 1986, 108, 625-629. [CrossRef]

57. Fokin, A.V.; Studnev, Y.N.; Rapkin, A.I.; Sultanbekov, D.A.; Potarina, T.M. Reaction of 1,1,2-trifluoro2-chloroethyldiethylamine with fluorocarboxylic acids. Bull. Acad. Sci. USSR Div. Chem. Sci. 1984, 33, 372-375. [CrossRef]

58. Cox, D.G.; Sprague, L.G.; Burton, D.J. The Facile Preparation of HF Free Polyfluorinated Acyl Fluorides. J. Fluorine Chem. 1983, 23, 383-388. [CrossRef]

59. Haas, A.; Plümer, R.; Schiller, A. Fluorierung ungesättigter Aldehyde mit Schwefeltetrafluorid. Chem. Ber. 1985, 118, 3004-3010. [CrossRef]

60. Anderson, G.L.; Burks, W.A.; Harruna, I.I. Novel Synthesis of 3-Fluoro-1-Aminoadamantane and Some of its Derivatives. Synth. Commun. 1988, 18, 1967-1974. [CrossRef]

61. Suzuki, M.; Nishida, Y.; Ohguro, Y.; Miura, Y.; Tsuchida, A.; Kobayashi, K. Synthesis and characterization of asymmetric $o$ - and $m$-nitrobenzoic acids with a 1,3-benzodioxole skeleton. Tetrahedron Asymmetry 2004, 15, 159-165. [CrossRef]

62. Kitazume, T.; Ishikawa, N. A Convenient synthesis of acetylenic ketones from $\beta$-diketones using $\alpha, \alpha$-difluoroalkylamines and freeze-dried potassium fluoride. Chem. Lett. 1980, 9, 1327-1328. [CrossRef]

63. Grieco, L.M.; Halliday, G.A.; Junk, C.P.; Lustig, S.R.; Marshall, W.J.; Petrov, V.A. Reactions of 1,1,2,2-tetrafluoroethyl-N,N-dimethylamine with linear and cyclic 1,3-diketones. J. Fluorine Chem. 2011, 132, 1198-1206. [CrossRef]

64. Autrey, R.L.; Scullard, P.W. The Second-Order Beckmann Reaction of an $\alpha$-(Methylthio) Ketone Oxime. J. Am. Chem. Soc. 1965, 87, 3284-3285. [CrossRef]

65. Autrey, R.L.; Scullard, P.W. Beckmann fragmentation of an $\alpha$-methylthio ketoxime. J. Am. Chem. Soc. 1968, 90, 4924-4929. [CrossRef]

66. Wakselman, C.; Tordeux, M. Acylation of electron-rich aromatic nucleus with fluorinated immonium salts. J. Chem. Soc. Chem. Commun. 1975, 956. [CrossRef]

67. Schmitt, E.; Rugeri, B.; Panossian, A.; Vors, J.P.; Pazenok, S.; Leroux, F.R. In Situ Generated Fluorinated Iminium Salts for Difluoromethylation and Difluoroacetylation. Org. Lett. 2015, 17, 4510-4513. [CrossRef] [PubMed] 
68. Watanabe, S.; Fujita, T.; Sakamoto, M.; Kitazume, T. Reaction of alcohols with N,N-diethyl-1,1,2,3,3,3hexafluoropropylamine in the presence of diisopropylethylamine. J. Fluorine Chem. 1988, 39, 17-22. [CrossRef]

69. Watanabe, S.; Fujita, T.; Sakamoto, A.; Endo, H.; Kitazume, T. Reactions of nitro alcohols with N,N-diethyl1,1,2,3,3,3-hexafluoropropylamine. J. Fluorine Chem. 1988, 38, 243-248. [CrossRef]

70. Watanabe, S.; Fujita, T.; Usui, Y.; Kimura, Y.; Kitazume, T. Fluorination of Halogeno Alcohols with 1,1,2,3,3,3Hexafluoropropyl Diethylamine. J. Fluorine Chem. 1986, 31, 135-141. [CrossRef]

71. Takaoka, A.; Iwamoto, K.; Kitazume, T.; Ishikawa, N. Preparation of Benzoheterocycles Containing a Chlorofluoromethyl Group Using the Yarovenko Reagent. J. Fluorine Chem. 1979, 14, 421-428. [CrossRef]

72. Du, S.; Tian, Z.; Yang, D.; Li, X.; Li, H.; Jia, C.; Che, C.; Wang, M.; Qin, Z. Synthesis, Antifungal Activity and Structure-Activity Relationships of Novel 3-(Difluoromethyl)-1-methyl-1H-pyrazole-4-carboxylic Acid Amides. Molecules 2015, 20, 8395-8408. [CrossRef] [PubMed]

73. Leroux, P.; Gredt, M.; Leroch, M.; Walker, A.S. Exploring mechanisms of resistance to respiratory inhibitors in field strains of Botrytis cinerea, the causal agent of gray mold. Appl. Environ. Microbiol. 2010, 76, 6615-6630. [CrossRef] [PubMed]

74. Walter, H. Pyrazole Carboxamide Fungicides Inhibiting Succinate Dehydrogenase. In Bioactive Heterocyclic Compound Classes; Wiley-VCH Verlag GmbH \& Co. KGaA: Weinheim, Germany, 2012; pp. 175-193.

75. Sierotzki, H.; Scalliet, G. A review of current knowledge of resistance aspects for the next-generation succinate dehydrogenase inhibitor fungicides. Phytopathology 2013, 103, 880-887. [CrossRef] [PubMed]

76. Wu, Z.-B.; Zhou, X.; Ye, Y.-Q.; Wang, P.-Y.; Yang, S. Design, synthesis and insecticidal activities of novel 1-substituted-5-(trifluoromethyl)-1H-pyrazole-4-carboxamide derivatives. Chin. Chem. Lett. 2017, 2, 121-125. [CrossRef]

77. Veloukas, T.; Karaoglanidis, G.S. Biological activity of the succinate dehydrogenase inhibitor fluopyram against Botrytis cinerea and fungal baseline sensitivity. Pest Manag. Sci. 2012, 68, 858-864. [CrossRef] [PubMed]

78. Fustero, S.; Simón-Fuentes, A.; Delgado, O.; Román, R. Fluorinated Pyrazoles and Indazoles. In Fluorine in Heterocyclic Chemistry Volume 1: 5-Membered Heterocycles and Macrocycles; Nenajdenko, V., Ed.; Springer: Cham, Switzerland, 2014; pp. 279-321.

79. Pazenok, S.; Lui, N.; Neeff, A. Process for Preparing 3-Dihalomethylpyrazole-4-Carboxylic Acid Derivatives. WO2008022777, 17 April 2008.

80. Giornal, F.; Pazenok, S.; Rodefeld, L.; Lui, N.; Vors, J.P.; Leroux, F.R. Synthesis of diversely fluorinated pyrazoles as novel active agrochemical ingredients. J. Fluorine Chem. 2013, 152, 2-11. [CrossRef]

81. Jaunzems, J.; Braun, M. An Atom-Efficient Route to Ethyl 3-(difluoromethyl)-1-methyl-1H-pyrazole-4carboxylate (DFMMP)—A Key Building Block for a Novel Fungicide Family. Org. Process Res. Dev. 2014, 18, 1055-1059. [CrossRef]

82. Graneto, M.J.; Phillips, W.G. 3-Difluoromethyl Pyrazole Carboxamide Fungicides. WO9212970, 6 August 1992.

83. Nett, M.; Grote, T.; Lohmann, J.K.; Dietz, J.; Smidt, S.P.; Rack, M.; Zierke, T. Method for Producing Difluoromethyl-Substituted Pyrazole Compounds. WO2008152138, 13 June 2008.

84. Pazenok, S.; Lui, N.; Heinrich, J.D.; Wollner, T. Method for the Regioselective Synthesis of 1-Alkyl-3Haloalkyl-Pyroazole-4-Carboxylic Acid Derivatives. WO2009106230, 12 February 2009.

85. Pashkevich, K.I.; Saloutin, V.I.; Fomin, A.N.; Berenblit, V.V.; Plashkin, V.S.; Postovskii, I.Y. Fluoroalkyl-Containing Monopyrazoles and Bispyrazoles. Zh. Vses. Khim. Ova+ 1981, 26, 105-107.

86. Claire, P.P. K.; Coe, P.L.; Jones, C.J.; Mccleverty, J.A. 3,5-Bis(Trifluoromethyl)Pyrazole and Some N-Substituted Derivatives. J. Fluorine Chem. 1991, 51, 283-289. [CrossRef]

87. Threadgill, M.D.; Heer, A.K.; Jones, B.G. The Reaction of 1,1,1,5,5,5-Hexafluoropentane-2,4-Dione with Hydrazines-A Reinvestigation. J. Fluorine Chem. 1993, 65, 21-23. [CrossRef]

88. Sloop, J.C.; Bumgardner, C.L.; Loehle, W.D. Synthesis of fluorinated heterocycles. J. Fluorine Chem. 2002, 118, 135-147. [CrossRef]

89. Obermayer, D.; Glasnov, T.N.; Kappe, C.O. Microwave-Assisted and Continuous Flow Multistep Synthesis of 4-(Pyrazol-1-yl)carboxanilides. J. Org. Chem. 2011, 76, 6657-6669. [CrossRef] [PubMed]

90. Maspero, A.; Giovenzana, G.B.; Monticelli, D.; Tagliapietra, S.; Palmisano, G.; Penoni, A. Filling the gap: Chemistry of 3,5-bis(trifluoromethyl)-1H-pyrazoles. J. Fluorine Chem. 2012, 139, 53-57. [CrossRef] 
91. Pazenok, S.; Giornal, F.; Landelle, G.; Lui, N.; Vors, J.P.; Leroux, F.R. A New Life for an Old Reagent: Fluoroalkyl Amino Reagents as Efficient Tools for the Synthesis of Diversely Fluorinated Pyrazoles. Eur. J. Org. Chem. 2013, 2013, 4249-4253. [CrossRef]

92. Giornal, F.; Landelle, G.; Lui, N.; Vors, J.P.; Pazenok, S.; Leroux, F.R. A New Synthesis and Process Development of Bis(fluoroalkyl)pyrazoles As Novel Agrophores. Org. Process Res. Dev. 2014, 18, 1002-1009. [CrossRef]

93. Schmitt, E.; Landelle, G.; Vors, J.P.; Lui, N.; Pazenok, S.; Leroux, F.R. A General Approach towards NH-Pyrazoles That Bear Diverse Fluoroalkyl Groups by Means of Fluorinated Iminium Salts. Eur. J. Org. Chem. 2015, 2015, 6052-6060. [CrossRef]

94. Norris, T.; Colon-Cruz, R.; Ripin, D.H.B. New hydroxy-pyrazoline intermediates, subtle regio-selectivity and relative reaction rate variations observed during acid catalyzed and neutral pyrazole cyclization. Org. Biomol. Chem. 2005, 3, 1844-1849. [CrossRef] [PubMed]

95. Fustero, S.; Roman, R.; Sanz-Cervera, J.F.; Simon-Fuentes, A.; Bueno, J.; Villanova, S. Synthesis of New Fluorinated Tebufenpyrad Analogs with Acaricidal Activity Through Regioselective Pyrazole Formation. J. Org. Chem. 2008, 73, 8545-8552. [CrossRef] [PubMed]

96. Bonacorso, H.G.; Porte, L.M.F.; Cechinel, C.A.; Paim, G.R.; Deon, E.D.; Zanatta, N.; Martins, M.A.P. DAST promotes the synthesis of new 5-(trifluoromethyl)-3-(1,1-difluoroethan-2-yl)-1H-pyrazoles. Tetrahedron Lett. 2009, 50, 1392-1394. [CrossRef]

97. Volochnyuk, D.M.; Pushechnikov, A.O.; Krotko, D.G.; Sibgatulin, D.A.; Kovalyova, S.A.; Tolmachev, A.A. Electron-rich amino heterocycles for regiospecific synthesis of trifluoro-methyl-containing fused pyridines. Synthesis 2003, 2003, 1531-1540. [CrossRef]

98. Boltacheva, N.S.; Filyakova, V.I.; Charushin, V.N. Fluoroalkyl-containing lithium 1,3-diketonates in reactions with amines and ammonium salts. Russ. J. Org. Chem. 2005, 41, 1452-1457. [CrossRef]

99. Mormino, M.G.; Fier, P.S.; Hartwig, J.F. Copper-Mediated Perfluoroalkylation of Heteroaryl Bromides with (phen)CuR F. Org. Lett. 2014, 16, 1744-1747. [CrossRef] [PubMed]

100. Duda, B.; Tverdomed, S.N.; Bassil, B.S.; Roschenthaler, G.V. Synthesis of highly substituted quinolines via heterocyclization of fluorinated acetylenephosphonates with ortho-aminoaryl ketones. Tetrahedron 2014, 70 , 8084-8096. [CrossRef]

101. Aribi, F.; Schmitt, E.; Panossian, A.; Vors, J.-P.; Pazenok, S.; Leroux, F.R. A new approach toward the synthesis of 2,4-bis(fluoroalkyl)-substituted quinoline derivatives using fluoroalkyl amino reagent chemistry. Org. Chem. Front. 2016, 3, 1392-1415. [CrossRef]

102. Ogura, K.; Ogu, K.-i.; Ayabe, T.; Sonehara, J.-I.; Akazome, M. Stereoselective formation of $\alpha$-fluoro- $\alpha$ trifluoromethyl- $\gamma$-lactones starting from $\gamma$-hydroxy- $\alpha, \beta$-unsaturated sulfones and a hexafluoropropenediethylamine adduct (PPDA). Tetrahedron Lett. 1997, 38, 5173-5176. [CrossRef]

103. Ogu, K.-i.; Akazome, M.; Ogura, K. A novel reaction of allylic alcohols with hexafluoropropene-diethylamine adduct (PPDA) to form 2-fluoro-2-trifluoromethyl-4-alkenamide. J. Fluorine Chem. 2003, 124, 69-80. [CrossRef]

104. Ogu, K.; Akazome, M.; Ogura, K. Diastereoselective formation of 2-fluoro-2-trifluoromethyl-3,4-alkadienamides from propargyl alcohols and hexafluoropropene-diethylamine adduct (PPDA). J. Fluorine Chem. 2004, 125, 429-438. [CrossRef]

105. Ogu, K.; Matsumoto, S.; Akazome, M.; Ogura, K. Novel Synthesis of $\alpha$-Trifluoromethylated $\alpha$-Amino Acid Derivatives from $\gamma$-Hydroxy- $\alpha$-fluoro- $\alpha$-trifluoromethyl Carboxamides. Org. Lett. 2005, 7, 589-592. [CrossRef] [PubMed]

106. Takaoka, A.; Ibrahim, M.K.; Kagaruki, S.R.F.; Ishikawa, N. Synthesis of Monofluoro Heterocycles Using Fluoroolefins as Starting Materials. Nippon Kagaku Kaishi 1985, 1985, 2169-2176. [CrossRef]

(C) 2017 by the authors. Licensee MDPI, Basel, Switzerland. This article is an open access article distributed under the terms and conditions of the Creative Commons Attribution (CC BY) license (http://creativecommons.org/licenses/by/4.0/). 